NBER WORKING PAPER SERIES

\title{
CAN STANDARD PREFERENCES EXPLAIN THE PRICES OF OUT OF THE MONEY S\&P 500 PUT OPTIONS
}

\author{
Luca Benzoni \\ Pierre-Collin Dufresne \\ Robert S. Goldstein \\ Working Paper 11861 \\ http://www.nber.org/papers/w11861
}

\author{
NATIONAL BUREAU OF ECONOMIC RESEARCH \\ 1050 Massachusetts Avenue \\ Cambridge, MA 02138 \\ December 2005
}

\begin{abstract}
We thank Raj Aggarwal, Gordon Alexander, Nicolae G^arleanu, Jun Liu, Jun Pan, Mark Rubinstein, and Tan Wang for helpful comments and suggestions. All errors remain our sole responsibility. The most recent version of this paper can be downloaded from http://www.umn.edu/ lbenzoni. The views expressed herein are those of the author(s) and do not necessarily reflect the views of the National Bureau of Economic Research.

(C)2005 by Luca Benzoni, Pierre-Collin Dufresne, and Robert S. Goldstein. All rights reserved. Short sections of text, not to exceed two paragraphs, may be quoted without explicit permission provided that full credit, including (C) notice, is given to the source.
\end{abstract}


Can Standard Preferences Explain the Prices of out of the Money S\&P 500 Put Options

Luca Benzoni, Pierre-Collin Dufresne, and Robert S. Goldstein

NBER Working Paper No. 11861

December 2005

JEL No. G21, G28, P51

\begin{abstract}
$\underline{\text { ABSTRACT }}$
Prior to the stock market crash of 1987, Black-Scholes implied volatilities of S\&P 500 index options were relatively constant across moneyness. Since the crash, however, deep out-of-the-money S\&P 500 put options have become 'expensive' relative to the Black-Scholes benchmark. Many researchers (e.g., Liu, Pan and Wang (2005)) have argued that such prices cannot be justified in a general equilibrium setting if the representative agent has 'standard preferences' and the endowment is an i.i.d. process. Below, however, we use the insight of Bansal and Yaron (2004) to demonstrate that the 'volatility smirk' can be rationalized if the agent is endowed with Epstein-Zin preferences and if the aggregate dividend and consumption processes are driven by a persistent stochastic growth variable that can jump. We identify a realistic calibration of the model that simultaneously matches the empirical properties of dividends, the equity premium, the prices of both at-the-money and deep out-of-the-money puts, and the level of the risk-free rate. A more challenging question (that to our knowledge has not been previously investigated) is whether one can explain within a standard preference framework the stark regime change in the volatility smirk that has maintained since the 1987 market crash. To this end, we extend the model to a Bayesian setting in which the agent updates her beliefs about the average jump size in the event of a jump. Note that such beliefs only update at crash dates, and hence can explain why the volatility smirk has not diminished over the last eighteen years. We find that the model can capture the shape of the implied volatility curve both preand post-crash while maintaining reasonable estimates for expected returns, price-dividend ratios, and risk-free rates.
\end{abstract}

Luca Benzoni

University of Minnesota

Carlson School of Management

321 19th Avenue South

Minneapolis, MN 55455

lbenzoni@csom.umn.edu

Pierre Collin-Dufresne

Haas School of Business F628

University of California -Berkeley

545 Student Services Bldg \#1900

Berkeley, CA 94720-1900

and NBER

deufresne@haas.berkeley.edu
Robert S. Goldstein

University of Minnesota

Finance Department

3-125 Carlson School of Management

321 19th Avenue South

Minneapolis, MN 55455

and NBER

rgoldstein@csom.umn.edu 


\section{Introduction}

In recent years, option prices on the S\&P 500 index (e.g., the SPX contract) have been the focus of much attention. One finding that researchers generally agree upon is that, prior to the 1987 stock market crash, implied 'volatility smiles' were relatively flat (see, e.g., Bates (2000)). However, since the crash, the Black-Scholes (B/S) formula has been producing systematic biases across 'moneyness' and maturity of SPX options. In particular, the B/S formula has been significantly underpricing short maturity, deep out-of-the-money puts. This property has been referred to as a 'volatility smirk' (see, e.g., Jackwerth and Rubinstein (1996) and Rubinstein (1994)).

Given the empirical failures of the B/S model in post-crash option data, much research has gone into identifying which assumptions of that model do not hold in practice. Focusing solely on stock returns, several authors have documented that, in contrast to the assumptions of B/S, stock prices jump and are subject to stochastic volatility. ${ }^{1}$ As such, new option pricing models have been proposed that incorporate these features (see, e.g., Bates (1996), Duffie et al. (2000), Heston (1993)). Furthermore, these extended models have been tested empirically. Among recent contributions, Bakshi et al. (1997, 2000), Bates (2000), and Huang and Wu (2004) extract information about the model parameters of the underlying returns process from derivatives prices alone. Benzoni (2002), Broadie et al. (2004), Chernov and Ghysels (2000), Eraker (2004), Jones (2003), and Pan (2002) use data on both underlying and derivatives prices to fit the model. Overall, these studies concur that a model with stochastic volatility and jumps significantly reduces the pricing and hedging errors of the Black-Scholes model, both in- and out-of-sample. ${ }^{2}$

These previous studies, however, focus on post-1987 option data. Further, they follow a partial equilibrium approach and let statistical evidence guide the exogenous specification of the underlying return dynamics. Such an approach leaves open two important questions:

- Does there exist a reasonable model for the dividend process and the preferences of agents that can explain the post-1987 SPX prices within a general equilibrium framework?

- Can such a model also explain the stark regime change in the volatility smirk that has maintained since the crash?

\footnotetext{
${ }^{1}$ See, e.g., Andersen et al. (2002), Chacko and Viceira (2003), Chernov et al. (1999, 2003), and Eraker et al. (2003).

${ }^{2} \mathrm{~A}$ related literature investigates the profits of option trading strategies (e.g., Coval and Shumway (2001) and Santa-Clara and Saretto (2004)) and the economic benefits of giving investors access to derivatives when they solve the portfolio choice problem (e.g., Constantinides et al. (2004), Driessen and Maenhout (2004) and Liu and Pan (2003)). Overall, these papers suggest that derivatives are non-redundant securities and, in particular, that volatility risk is priced. These findings are consistent with the evidence in Bakshi and Kapadia (2003) and Buraschi and Jackwerth (2001), as well as with the results of the studies that use data on both underlying and derivatives prices to fit parametric stochastic volatility models.
} 
These questions are the focus of this paper. Interestingly, while the first question has already received considerable attention in the literature, the second question, to our knowledge, has been largely ignored.

Regarding the first question, the general consensus of the existing literature seems to be that it is difficult to reconcile the post-crash prices of short-maturity, deep out-of-the-money put options within a general equilibrium setting when the representative agent has standard expected utility preferences. For example, the problem with specifying a constant relative risk-aversion utility function for the representative agent is that the risk-aversion parameter $\gamma$ is forced to govern both the premium on equity and out-of-the-money puts. Liu, Pan and Wang (2004, LPW) consider a model in which the endowment is an i.i.d. process and demonstrate that in this setting such restriction is inconsistent with the empirical evidence presented in, e.g., Jackwerth (2000), Pan (2002), and Rosenberg and Engle (2002). Further, LPW find that even the two-parameter recursive utility of Epstein-Zin (1989) and Kreps-Porteus (1978) cannot explain the puzzle within their framework, since it generates results very similar to those of a standard power utility setting (see, e.g., page 151 of LPW).

Consistent with these empirical findings, much of the literature has advocated alternative preferences outside of the standard state-independent expected utility framework (see, e.g., Bates (2001), Brown and Jackwerth (2004), and LPW). ${ }^{3}$ For example, LPW view the option smirk as evidence for non-expected utility preferences on the part of market participants. Specifically, they argue that if the endowment is an i.i.d. process, then in order to reconcile the prices of options and the underlying index, agents must exhibit 'uncertainty aversion' towards rare events that is different from the standard 'risk-aversion' they exhibit towards diffusive risk. In effect, they provide a decision-theoretic basis to the idea of crash aversion advocated by Bates (2001), who proposed an ad-hoc extension of the standard power utility that allows for a special risk-adjustment parameter for jump risk distinct from that for diffusive risk.

In this paper, however, we take a different approach. In particular, we expand upon the insights of Bansal and Yaron (2004, BY) by considering Epstein and Zin (1989) preferences and specifying the expected growth rate of dividends to be driven by a persistent stochastic variable that follows a

\footnotetext{
${ }^{3}$ Bates (2001) proposes a model in which agents exhibit special crash aversion to capture many stylized facts from stock index options markets. Brown and Jackwerth (2004) consider a representative agent model in which the marginal utility of the representative agent is driven by a second state variable that is a function of a 'momentum' state variable. Related, Bondarenko (2003) argues that in order to explain S\&P 500 put prices a candidate equilibrium model must produce a path-dependent projected pricing kernel. Finally, Buraschi and Jiltsov (2005) consider a model in which heterogeneity in beliefs over the dividend growth rate generates state dependent utility. They focus on the volume of trading in the option market.
} 
jump-diffusion process. This approach contrasts with that of LPW and Bates (2001), who consider non-standard preferences and specify dividends as an i.i.d. jump-diffusion process with constant drift and volatility. However, as noted by BY and Shephard and Harvey (1990), it is very difficult to distinguish between a purely i.i.d. process and one which incorporates a small persistent component. Nevertheless, the presence of a small persistent component can have important asset pricing implications.

Our approach has several desirable features. First, consistent with observation, our model predicts that the dividend process is relatively smooth during a crash event, in contrast to these previous papers that can only capture a $23 \%$ market crash with a $23 \%$ drop in dividends. Second, our model is consistent with a large drop in the risk-free rate on crash dates, unlike these previous papers that predict a constant risk-free rate. Finally, in contrast to Brown and Jackwerth (2004), by incorporating jumps in the underlying processes we can capture the volatility smirk with a model calibration that is more consistent with observation.

Within this framework, we show that a model with standard preferences and a realistic calibration of the aggregate endowment process can simultaneously capture the levels of the equity risk premium and the risk-free rate, and the prices of deep out-of-the-money put options. The intuition for these results is similar to that discussed in BY. ${ }^{4}$ Epstein and Zin preferences allow for a separation of the elasticity of intertemporal substitution (EIS) and risk aversion. When the EIS is larger than one, the intertemporal substitution effect dominates the wealth effect. Thus, in response to higher expected growth agents buy more assets, and consequently prices increase. The opposite occurs when there is a decrease in expected growth, e.g., because of an unexpected downward jump in the predictable component of dividends that triggers a market crash. In this framework, the risky asset exhibits positive returns when the state is good, while it performs poorly in the bad state. As such, investors demand a high equity premium and are willing to pay a high price for a security that delivers insurance in the bad state, like, e.g., a put option on the S\&P 500 index.

To maintain parsimony, we follow LPW and restrict our dividend dynamics to have constant volatility. That is, we consider only the so-called 'one-channel' BY case. Extending the model to incorporate stochastic volatility (the 'two-channel' BY case) is straightforward, but in order to keep the model parsimonious, we choose to focus on a rather minimal model. We solve the model

\footnotetext{
${ }^{4}$ We emphasize, however, that most existing models that capture the equity premium (e.g., BY, Bansal et al. (2004), and Campbell and Cochrane (1999)) specify the dividend growth rate process as continuous. As such, these models cannot account for the high premium on near-term out-of-the-money put options, nor the possibility of a market crash.
} 
using standard results in recursive utility (e.g., Duffie and Epstein (1992a,b), Duffie and Skiadas (1994), Schroder and Skiadas (1999, 2003), and Skiadas (2003)). We show that the price-dividend ratio satisfies an integro-differential equation that is non-linear when the EIS is different from the inverse of the coefficient of risk aversion. To solve such equations, we use the approximation method of Collin-Dufresne and Goldstein (2005), which is itself an extension of the Campbell-Shiller approximation (see Campbell and Shiller (1988)).

We consider a realistic calibration of the model and we illustrate its implications for the pricing of short-maturity SPX options. In our baseline case, a put option with maturity of one month and a strike price that is $10 \%$ out-of-the-money has an implied volatility of approximately $24 \%$. In contrast, a one-month, at-the-money option has an implied volatility of approximately $14 \%$. That is, consistent with empirical evidence, we find a $10 \%$ volatility smirk. Further, we find that our baseline case also captures the empirical properties of dividends and consumption, and generates a realistic $1 \%$ real risk-free rate, a $6 \%$ equity premium, and a price-dividend ratio of 20 . Sensitivity analysis is performed suggesting that the main qualitative results are robust to a wide range of parameter calibrations. In sum, we conclude that our specification successfully captures many salient properties of the SPX prices during the post-1987 period.

Next, we proceed to investigate the second question of the paper. In particular, we examine whether our model can also explain the stark change in the implied volatility pattern that has been observed since the 1987 market crash. We note that an extreme event such as the 1987 crash is likely to dramatically change the investor's perception about the nature of possible future market fluctuations. To formalize this intuition, we extend the model to a Bayesian setting in which the agent formulates a prior on the average value of the jump size, and then updates her prior when she observes an extreme event such as the 1987 crash. Note that the updating of beliefs only occurs at crash dates. As such, her posterior beliefs on the average value of the jump size are potentially very long lived, and hence can explain why the volatility smirk has remained high even eighteen years after the crash.

We find that our model can capture the implied volatility pattern of option prices both before and after the 1987 crash. Specifically, we present simulation results in which the steepness of the volatility smirk (i.e., the difference between implied volatilities of $10 \%$-out-of-the-money and at-themoney puts) is approximately $3 \%$, a number that is consistent with the pre-crash evidence reported in, e.g., Bates (2000). At the same time, the occurrence of a jump triggers the updating of the agent's beliefs about the expected value of the jump size. As such, after the crash, out-of-the-money put options are perceived to be more valuable, and the volatility smirk becomes as steep as $10 \%$. 
Furthermore, consistent with observation the model predicts a downward jump in the risk-free rate during crash events.

One failure of our model, however, is its predictions for the day of the crash. In particular, our model predicts jumps in both the stock price and risk-free rate that are even more extreme than what was observed in the 1987 crash. We attribute this failure to our choice of keeping the model as parsimonious as possible, and we suppose that if additional parameters and/or state variables were added, we could further improve the fit. In addition, we note that there are institutional features that may have attenuated the fluctuation in interest rates and market prices during the crash day. $^{5}$

In focusing on the pricing of both out-of-the-money put options and the equity index, our paper is related to the recent literature that searches for a pricing kernel derived within a general equilibrium setting that can simultaneously capture the salient features of equity returns, riskfree rates, and the prices of derivative securities. For example, Chen et al. (2004) investigate the ability of the BY and Campbell and Cochrane (1999) models to jointly price equity and risky (defaultable) corporate debt. Bansal et al. (2004) examine the implications of the BY and Campbell and Cochrane (1999) models for the pricing of at-the-money options on a stock market index as well as on consumption and wealth claims. ${ }^{6}$

Also related is a growing literature that investigates the effect of changes in investors' sentiment (e.g., Han (2005)), market structure, and net buying pressure (e.g., Bollen and Whaley (2004), Dennis and Mayhew (2002), and Gârleanu et al. (2005)) on the shape of the implied volatility smile. ${ }^{7}$ These papers, however, do not address why end users buy these options at high prices

\footnotetext{
${ }^{5}$ On October 19 and 20, 1987, the S\&P 500 Futures price was considerably lower than the index price, which suggests that the drop in the index level does not fully represent the magnitude of the market adjustment in prices. This evidence can be explained by the existence of significant delays in the submission and execution of limit orders during the crash events, magnified by the standard problem of 'stale' prices (see, e.g., Kleidon (1992)). Moreover, interventions of the exchange might have further contained the fluctuations in stock prices during the crash. Finally, the Fed assured that it would provide adequate liquidity to the U.S. financial system necessary to calm the equity and other markets (see, e.g., p. 3 of the November 3, 1987, 'Notes for FOMC Meeting' document available from the Federal Reserve web site http://www.federalreserve.gov/fomc/transcripts/1987/871103StaffState.pdf).

${ }^{6}$ Other papers that examine the asset pricing implications of the BY model include Bansal and Lundblad (2002), Hansen, Heaton, and Li (2004), and Malloy et al. (2005).

${ }^{7}$ This literature argues that due to the existence of limits to arbitrage, market makers cannot always fully hedge their positions (see, e.g., Green and Figlewski (1999), Figlewski (1989), Hugonnier et al. (2005), Liu and Longstaff (2004), Longstaff (1995), and Shleifer and Vishny (1997)). As such, they are likely to charge higher prices when asked to absorb large positions in certain option contracts. Consistent with this view, Han (2005) finds that the S\&P 500 option volatility smile tends to be steeper when survey evidence suggests that investors are more bearish, when large speculators hold more negative net positions in the S\&P 500 index futures, and when the index level drops relative to its fundamentals. Related, Bollen and Whaley (2004) and Gârleanu et al. (2005) identify an excess of buyer-motivated trades in out-of-the-money SPX puts and find a positive link between demand pressure and the steepness of the volatility smirk.
} 
relative to the $\mathrm{B} / \mathrm{S}$ value or why the 1987 crash changed the shape of the volatility smile so dramatically and permanently. Our paper offers one possible explanation.

The rest of the paper is organized as follows. In Section 2, we present an option pricing model that explains the post-1987 volatility smirk in SPX prices. In Section 3, we extend our setting to incorporate Bayesian updating of the agent's believes. We use this setting to show that an event such as the 1987 market crash can generate a change in the SPX price that is qualitatively consistent with what we observe in the data. We conclude in Section 4.

\section{A General Equilibrium Model of the Volatility Smirk}

In this section, we present a general equilibrium model that produces option prices that are consistent with the post-1987 evidence. We specify the consumption and dividend dynamics as

$$
\begin{aligned}
\frac{d C}{C} & =\left(\mu_{C}+x\right) d t+\sqrt{\Omega} d z_{C} \\
\frac{d D}{D} & =\left(\mu_{D}+\phi x\right) d t+\sigma_{D} \sqrt{\Omega}\left(\rho_{C, D} d z_{C}+\sqrt{1-\rho_{C, D}^{2}} d z_{D}\right) \\
d x & =-\kappa_{x} x d t+\sigma_{x} \sqrt{\Omega} d z_{x}+\widetilde{\nu} d N .
\end{aligned}
$$

Here, $\left\{d z_{C}, d z_{D}, d z_{x}\right\}$ are uncorrelated Brownian motions, the Poisson jump process $d N$ has a jump intensity equal to $\lambda$ and the jump size $\widetilde{\nu}$ is normally distributed:

$$
\begin{aligned}
\mathrm{E}[d N] & =\lambda d t \\
\widetilde{\nu} & \leadsto N\left(\mu_{\nu}, \sigma_{\nu}\right) .
\end{aligned}
$$

It is convenient to define $c \equiv \log C$ and $\delta \equiv \log D$. Itô's formula then yields

$$
\begin{aligned}
d c & =\left(\mu_{C}+x-\frac{1}{2} \Omega\right) d t+\sqrt{\Omega} d z_{C} \\
d \delta & =\left(\mu_{D}+\phi x-\frac{1}{2} \sigma_{D}^{2} \Omega\right) d t+\sigma_{D} \sqrt{\Omega}\left(\rho_{C, D} d z_{C}+\sqrt{1-\rho_{C, D}^{2}} d z_{D}\right) .
\end{aligned}
$$

We note that our specification is similar to the so-called one-channel BY model, in which the expected growth rates in dividend and consumption are stochastic. There is however one important difference - in our setting, the state variable driving the expected growth rate in consumption and dividend (i.e., the $x$ process) is subject to jumps. Consistent with BY, we calibrate the mean reversion parameter $\kappa_{x}$ to be relatively low, implying that the effect of a downward jump may be very long-lived. As we demonstrate below, this persistence causes the agent in our model to be willing to pay a high premium to buy out-of-the-money put options in order to hedge downside risk. 
In contrast to our model, LPW assume that the dividend growth rate is subject to jumps, while the expected dividend growth rate is constant in their model. That is, in their model a crash is due to a downward jump in the dividend level. ${ }^{8}$ In particular, in order to explain a $23 \%$ drop in market prices, their model predicts that the dividend also drops by $23 \%$. We note that such large jumps are not observed in the dividend data, which are instead relatively smooth.

\subsection{Recursive Utility}

Following Epstein and Zin (1989), we assume that the representative agent's preferences over a consumption process $\left\{C_{t}\right\}$ are represented by a utility index $U(t)$ that satisfies the following recursive equation:

$$
U(t)=\left\{\left(1-e^{-\beta d t}\right) C_{t}^{1-\rho}+e^{-\beta d t} \mathrm{E}_{t}\left(U(t+d t)^{1-\gamma}\right)^{\frac{1-\rho}{1-\gamma}}\right\}^{\frac{1}{1-\rho}} .
$$

With $d t=1$, this is the discrete time formulation of Kreps-Porteus/Epstein-Zin (KPEZ), in which $\Psi \equiv 1 / \rho$ is the EIS and $\gamma$ is the risk-aversion coefficient.

The properties of the stochastic differential utility in (8) and the related implications for asset pricing have been previously studied by, e.g., Duffie and Epstein (1992a,b), Duffie and Skiadas (1994), Schroder and Skiadas (1999, 2003), and Skiadas (2003). In Appendix A, we extend their results to the case in which the aggregate output has jump-diffusion dynamics. ${ }^{9}$ The solution to our model is a special case of such general results and follows immediately from Propositions 1 and 2 in Appendix A. Specifically, when $\rho, \gamma \neq 1$ Proposition 1 gives the agent's value function as:

$$
J=\frac{e^{c(1-\gamma)}}{1-\gamma} \beta^{\theta} I(x)^{\theta}
$$

where $I$ denotes the price-consumption ratio and satisfies the following equation

$$
\begin{array}{r}
0=I\left[(1-\gamma) \mu_{C}+(1-\gamma) x-\frac{\gamma}{2}(1-\gamma) \Omega-\beta \theta\right]-\kappa_{x} x \theta I_{x} \\
+\frac{1}{2} \sigma_{x}^{2} \Omega \theta\left[(\theta-1)\left(\frac{I_{x}}{I}\right)^{2} I+I_{x x}\right]+\lambda I \mathcal{J} I^{\theta}+\theta,
\end{array}
$$

and where we have defined the operator

$$
\mathcal{J} h(x)=\mathrm{E}\left[\frac{h(x+\nu)}{h(x)}\right]-1
$$

\footnotetext{
${ }^{8}$ Barro (2005) and Bates (2001) make a similar assumption about the dividend dynamics in their model.

${ }^{9} \mathrm{~A}$ related literature studies the general equilibrium properties of a jump-diffusion economy in which the agent has non-recursive utility; see, e.g., Ahn and Thompson (1988) and Naik and Lee (1990). Also related, Cvitanić et al. (2005) and Liu, Longstaff, and Pan (2003) examine the optimal portfolio choice problem when asset returns (or their volatility) are subject to jumps.
} 
To obtain an approximate solution for $I(x)$, we use the method of Collin-Dufresne and Goldstein (2005), which itself is in the spirit of the Campbell-Shiller approximation. In particular, we note that $I(x)$ would possess an exponential affine solution if the last term on the right-hand-side (RHS) of equation (10) (the $\theta$ term) were absent. As such, we move $\theta$ to the left-hand-side (LHS) and then add to both sides of the equation the term $h(x) \equiv\left(n_{0}+n_{1} x\right) e^{A+B x}$. Hence, we re-write equation (10) as

$$
\begin{aligned}
\left(n_{0}+n_{1} x\right) e^{A+B x}-\theta= & \left(n_{0}+n_{1} x\right) e^{A+B x} \\
& +I\left[(1-\gamma) \mu_{C}+(1-\gamma) x-\frac{\gamma}{2}(1-\gamma) \Omega-\beta \theta\right]-\kappa_{x} x \theta I_{x} \\
& +\frac{1}{2} \sigma_{x}^{2} \Omega \theta\left[(\theta-1)\left(\frac{I_{x}}{I}\right)^{2} I+I_{x x}\right]+\lambda I \mathcal{J} I^{\theta} .
\end{aligned}
$$

We then approximate the RHS to be identically zero and look for a solution of the form

$$
I(x)=e^{A+B x} .
$$

We find this form to be self-consistent in that the only terms that show up are either linear in or independent of $x$. This approach provides us with two equations, which we interpret as identifying the $\left\{n_{0}, n_{1}\right\}$ coefficients in terms of $B$

$$
\begin{aligned}
-n_{0} & =(1-\gamma) \mu_{C}-\frac{\gamma}{2}(1-\gamma) \Omega-\beta \theta+\frac{1}{2} \sigma_{x}^{2} \Omega(\theta B)^{2}+\lambda\left(\chi_{\theta B}^{P}-1\right) \\
-n_{1} & =(1-\gamma)-\kappa_{x} \theta B
\end{aligned}
$$

where we have defined

$$
\chi_{a}^{P} \equiv \mathrm{E}\left[e^{a \widetilde{\nu}}\right]=e^{a \mu_{\nu}+\frac{1}{2} a^{2} \sigma_{\nu}^{2}} .
$$

Note that our solution is specified once we identify the four parameters $\left\{A, B, n_{0}, n_{1}\right\}$. To this end, the system (14)-(15) provides two identifying conditions. The last two equations necessary to identify the remaining parameters are obtained from minimizing the following unconditional expectation:

$$
\min _{\{A, B\}} \mathrm{E}_{-\infty}\left\{(L H S)^{2}\right\}=\min _{\{A, B\}} \mathrm{E}_{-\infty}\left\{\left(\left(n_{0}+n_{1} x\right) e^{A+B x}-\theta\right)^{2}\right\} .
$$

The logic of this condition is as follows. Recall that we have set the RHS to zero above. Here, we are choosing the parameters so that the LHS is as close to zero as possible (in a least-squares error metric). Collin-Dufresne and Goldstein (2005) show that this approach provides a very accurate approximation to the problem solution. 
We note that the Campbell-Shiller approximation is similar in that their first two equations are as in (14)-(15) above. However, their last two equations satisfy ${ }^{10}$

$$
\begin{aligned}
0 & =\left.[L H S(x)]\right|_{x=\mathrm{E}_{-\infty}[x]} \\
0 & =\left.\frac{\partial}{\partial x}[\operatorname{LHS}(x)]\right|_{x=\mathrm{E}_{-\infty}[x]} .
\end{aligned}
$$

\section{$2.2 \quad$ Risk-Free Rate and Risk-Neutral Dynamics}

When $\rho, \gamma \neq 1$, Proposition 1 in Appendix A gives the pricing kernel as

$$
\Pi(t)=e^{\int_{0}^{t} d s\left((\theta-1) I\left(x_{s}\right)^{-1}-\beta \theta\right)} \beta^{\theta} e^{-\gamma c_{t}} I\left(x_{t}\right)^{\theta-1},
$$

which has dynamics

$$
\frac{d \Pi}{\Pi}=-r d t-\gamma \sqrt{\Omega} d z_{C}+(\theta-1) B \sigma_{x} \sqrt{\Omega} d z_{x}+\left[\frac{I^{\theta-1}(x+\widetilde{\nu})}{I^{\theta-1}(x)}-1\right] d N-\lambda \mathcal{J} I(x)^{\theta-1} d t,
$$

where the risk-free rate $r$ is given by Proposition 2 in Appendix A $(\rho, \gamma \neq 1)$ :

$$
\begin{aligned}
r & =r_{0}+\rho x \\
r_{0} & \equiv \beta+\rho \mu_{C}-\frac{\gamma}{2} \Omega(1+\rho)-\sigma_{x}^{2} \Omega(1-\theta) \frac{B^{2}}{2}-\lambda\left(\chi_{(\theta-1) B}^{P}-1\right)+\frac{\theta-1}{\theta} \lambda\left(\chi_{\theta B}^{P}-1\right) .
\end{aligned}
$$

Given the pricing kernel dynamics, it is straightforward to determine the risk-neutral dynamics. We find

$$
\begin{aligned}
d c & =\left(\mu_{C}+x-\Omega\left(\frac{1}{2}+\gamma\right)\right) d t+\sqrt{\Omega} d z_{C}^{Q} \\
d \delta & =\left(\mu_{D}+\phi x-\sigma_{D} \Omega\left(\frac{1}{2} \sigma_{D}+\rho_{C, D} \gamma\right)\right) d t+\sigma_{D} \sqrt{\Omega}\left(\rho_{C, D} d z_{C}^{Q}+\sqrt{1-\rho_{C, D}^{2}} d z_{D}^{Q}\right) \\
d x & =\left(-\kappa_{x} x-(1-\theta) B \sigma_{x}^{2} \Omega\right) d t+\sigma_{x} \sqrt{\Omega} d z_{x}^{Q}+\widetilde{\nu} d N,
\end{aligned}
$$

where the three Brownian motions $\left\{d z_{C}^{Q}, d z_{x}^{Q}, d z_{\Omega}^{Q}\right\}$ are uncorrelated, and the Q-intensity of the Poisson jump process $N$ is

$$
\lambda^{Q}=\lambda \chi_{(\theta-1) B}^{P} .
$$

Furthermore, the Q-probability density of the jump amplitudes is

$$
\begin{aligned}
\pi^{Q}(\widetilde{\nu}=\nu) & =\pi(\widetilde{\nu}=\nu) \frac{I^{\theta-1}(x+\nu)}{\mathrm{E}\left[I^{\theta-1}(x+\widetilde{\nu})\right]} \\
& =\frac{1}{\sqrt{2 \pi \sigma_{\nu}^{2}}} \exp \left\{\left(-\frac{1}{2 \sigma_{\nu}^{2}}\right)\left[\nu-\mu_{\nu}-(\theta-1) B \sigma_{\nu}^{2}\right]^{2}\right\}
\end{aligned}
$$

\footnotetext{
${ }^{10}$ Note that $\mathrm{E}_{-\infty}[x] \neq 0$ since we have written the state vector dynamics without compensator terms on the jumps.
} 
That is,

$$
\begin{aligned}
\widetilde{\nu}^{Q} & \leadsto N\left(\mu_{\nu}^{Q}, \sigma_{\nu}\right) \\
\mu_{\nu}^{Q} & =\mu_{\nu}+(\theta-1) B \sigma_{\nu}^{2} .
\end{aligned}
$$

\subsection{Dividend Claim}

Define $V(D, x)$ as the claim to dividend. By construction, the expected return under the risk neutral measure is the risk-free rate:

$$
\mathrm{E}_{t}^{Q}\left[\frac{d V+D d t}{V}\right]=r d t
$$

It is convenient to define the price-divided ratio $I^{D} \equiv \frac{V}{D}$. Equation (28) can thus be written as

$$
\begin{aligned}
r-\frac{1}{I^{D}} & =\frac{1}{d t} \mathrm{E}^{Q}\left[\frac{d V}{V}\right] \\
& =\frac{1}{d t} \mathrm{E}^{Q}\left[\frac{d I^{D}}{I^{D}}+\frac{d D}{D}+\frac{d D}{D} \frac{d I^{D}}{I^{D}}\right]
\end{aligned}
$$

We look for a solution of the form

$$
I^{D}(x)=e^{F+G x}
$$

We use the risk-neutral dividend and $x$-dynamics (23)-(24) to re-write equation (29) as

$$
r-\frac{1}{I^{D}}=\mu_{D}+\phi x-\gamma \rho_{C, D} \sigma_{D} \Omega-\kappa_{x} x G-(1-\theta) B G \sigma_{x}^{2} \Omega+\frac{1}{2} G^{2} \sigma_{x}^{2} \Omega+\lambda^{Q}\left(\chi_{G}^{Q}-1\right),
$$

where we have defined

$$
\chi_{a}^{Q} \equiv \mathrm{E}^{Q}\left[e^{a \widetilde{\nu}}\right]=e^{a \mu_{\nu}^{Q}+\frac{1}{2} a^{2} \sigma_{\nu}^{2}}
$$

As above, we find an approximate solution for $I^{D}$ by moving $r$ to the RHS, multiplying both sides by $I^{D}$, and adding $\left(m_{0}+m_{1} x\right) I^{D}$ to both sides. These calculations give

$$
\begin{aligned}
L H S= & \left(m_{0}+m_{1} x\right) e^{F+G x}-1 \\
\left(\frac{1}{I^{D}}\right) R H S= & \left(m_{0}+m_{1} x\right)-r+\mu_{D}+\phi x-\gamma \rho_{C, D} \sigma_{D} \Omega-\kappa_{x} x G \\
& -(1-\theta) B G \sigma_{x}^{2} \Omega+\frac{1}{2} G^{2} \sigma_{x}^{2} \Omega+\lambda^{Q}\left(\chi_{G}^{Q}-1\right) .
\end{aligned}
$$

From equation (20), $r=r_{0}+\rho x$. Hence, if we approximate the RHS to be identically zero, and then collect terms linear in and independent of $x$, respectively, we obtain the system:

$$
\begin{aligned}
& -m_{0}=-r_{0}+\mu_{D}-\gamma \rho_{C, D} \sigma_{D} \Omega-(1-\theta) B G \sigma_{x}^{2} \Omega+\frac{1}{2} G^{2} \sigma_{x}^{2} \Omega+\lambda^{Q}\left(\chi_{G}^{Q}-1\right) \\
& -m_{1}=-\rho-\kappa_{x} G+\phi .
\end{aligned}
$$


Equations (35)-(36) should be interpreted as specifying the $\left\{m_{0}, m_{1}\right\}$ in terms of $G$. In turn, we identify $\{F, G\}$ by minimizing the unconditional squared error:

$$
\min _{\{F, G\}} \mathrm{E}_{-\infty}\left[(L H S)^{2}\right]=\min _{\{F, G\}} \mathrm{E}_{-\infty}\left[\left(\left(m_{0}+m_{1} x\right) e^{F+G x}-1\right)^{2}\right] .
$$

\subsection{The Equity Premium}

The general form of the risk premium on the risky asset is given in equation (102) of Proposition 2 in Appendix A. Here, such expression simplifies to

$$
\text { Equity Premium }=\gamma \sigma_{D} \rho_{C, D} \Omega+(1-\theta) B G \sigma_{x}^{2} \Omega-\lambda\left[\chi_{G+(\theta-1) B}^{P}-\chi_{G}^{P}-\chi_{(\theta-1) B}^{P}+1\right],
$$

where the transform $\chi^{P}$ was previously defined in equation (16).

In equation (38), the second and third terms represent the risk premia on the diffusive and jump components of expected growth risk. We note that in the constant relative risk aversion (CRRA) case, $\gamma$ equals $1 / \Psi$, and therefore $\theta=1$. As such, the last two terms in equation (38) vanish and the CRRA equity premium reduces to $\left(\gamma \sigma_{D} \rho_{C, D}\right)$. Thus, as in BY, with CRRA utility a persistent endowment process cannot generate a realistic equity premium, let alone explain out-of-the-money put prices.

On the other hand, in the KPEZ case with $\Psi>1$, the risk premium on expected growth risk is positive. As in BY, the mechanism for this results is as follows. When $\Psi>1$, the intertemporal substitution effect dominates the wealth effect. Thus, in response to higher expected growth, agents buy more assets, and consequently the wealth-to-consumption ratio increases. That is, in this scenario the coefficient $B$ in the wealth-to-consumption-ratio function (13) is positive. In addition, due to the effect of leverage the coefficient $G$ in the price-to-dividend ratio function (30) is larger than $B$. Hence, the last two terms in equation (38) are positive. Intuitively, with KPEZ utility and $\Psi>1$, the stock exhibits positive returns when the state is good, while it performs poorly in the bad state. As such, investors demand a higher risk premium.

\subsection{Valuing Options on the Dividend Claim}

The date- $t$ value of an European call option on the dividend claim $V_{t}=D_{t} e^{F+G x_{t}}$, with maturity $T$ and strike price $K$, is given by

$$
C\left(V_{t}, x_{t}, K, T\right)=\mathrm{E}_{t}^{Q}\left[e^{-\int_{t}^{T} r\left(x_{s}\right) d s}\left(V_{T}-K\right)^{+}\right] .
$$


We note that our model is affine. As such, the option pricing problem can be solved using standard inverse Fourier transform techniques (see, e.g., Bates (1996), Duffie et al. (2000), and Heston (1993)). In Appendix B, we report a semi-closed form formula for the price of an option given in equation (39).

\subsection{Model Calibration}

To illustrate the implications of the model, we consider a realistic calibration of its coefficients. In the next section, we will show that our main result is robust to a wide range of parameter calibrations.

\section{Consumption and Dividend Dynamics:}

To calibrate the consumption process in equations (6), we rely on the model coefficients reported in BY. BY use the convention to express their parameters in decimal form with monthly scaling. Here, instead, we express them in decimal form with yearly scaling. After adjusting for differences in scaling, we fix $\mu_{C}=0.018$ and $\Omega=0.00073$.

We note that corporate leverage justifies a higher expected growth rate in dividends than in consumption (see, e.g., Abel (1999)). This can be modeled by setting $\mu_{D}>\mu_{C}$ and $\phi>1$ in equation (7). As such, we fix $\mu_{D}=0.025$ and $\phi=1.5$. We note the difference with BY, who assume $\mu_{D}=\mu_{C}$ and model leverage entirely through the $\phi$ coefficient, which they choose to be in the 3-3.5 range. We use $\sigma_{D}=4.5$, the same value of BY. Finally, we allow for a $60 \%$ correlation between consumption and dividend, i.e., $\rho_{C, D}=0.6$.

In the $x$-dynamics (3), we use $\kappa_{x}=0.3$. This is in line with the value used by BY (if we adjust for differences in scaling and we map the $\mathrm{BY} \operatorname{AR}(1) \rho$ coefficient into the $\kappa_{x}$ of our continuous-time specification, we find $\kappa_{x}=0.2547$ ). We fix $\sigma_{x}=0.4472$, a value similar to, but slightly lower than that of BY (i.e., 0.5280, after adjusting for differences in scaling). A slightly lower value of $\sigma_{x}$ is justified by the fact that part of the variance of the $x$ process is driven by the jump component, which is absent in the BY model.

Finally, we calibrate the Poisson jump intensity process to yield, on average, one jump every fifty years, i.e., $\lambda=0.02$. This is consistent with the intuition that our jump process captures extreme and very rare price fluctuations such as the 1987 market crash. Further, we fix $\mu_{\nu}=-0.094$. This approach implies that one jump of average size produces a fall in market prices of approximately $23 \%$, which is in line with the $24.5 \%$ drop in the S\&P 500 index 
observed in between the close of Thursday, October 15, and Monday, October 19, 1987. Finally, we fix the standard deviation of the jump size to $\sigma_{\nu}=0.015$.

\section{Preferences:}

We use a time discount factor coefficient $\beta=0.023$.

Mehra and Prescott (1985) argue that reasonable values of the relative risk aversion coefficient $\gamma$ are smaller than 10. BY consider $\gamma=7.5$ and 10. Bansal et al. (2004) report $\gamma=7.1421$. As such, we fix $\gamma=7.5$ in our baseline case.

The magnitude of the coefficient $\rho$ is more controversial. Hall (1988) argues that the EIS is below 1. However, Attanasio and Weber (1989), Bansal et al. (2004), Guvenen (2001), Hansen and Singleton (1982), among others, estimate the EIS to be in excess of 1. In particular, Attanasio and Weber (1989) find estimates that are close to 2. Bansal et al. (2004) estimate the EIS to be in the 1.5-2.5 region, and fix it at 2 in their application. Here we follow Bansal et al. (2004) and use $\Psi=1 / \rho=2$ for our baseline case.

In the next section, we document the sensitivity of our results to different values of $\gamma$ and $\Psi$.

\section{Initial Conditions:}

In the plots below, we fix the state variable $x$ at its steady-state mean value $x_{0}=\mu_{\nu} \lambda / \kappa_{x}$. We note that $x_{0}$ is nearly zero in our calibration, i.e., it is very close to the steady-state mean value of $x$ in the BY model. Further, we emphasize that our results are robust to the value assigned to the state $x$. Specifically, when option prices are computed at values of $x$ that are within \pm 3 standard deviations from the steady-state mean $x_{0}$, we obtain implied volatility plots that are very similar to those reported below.

\subsection{Simulation Results}

We note that our calibration yields realistic values of the risk-free rate, the equity premium, and the price-dividend ratio. Specifically, in the baseline case we find that the steady-state real risk-free rate is $0.93 \%$, while the equity premium predicted by the model is $5.76 \%$. Further, we find that the steady-state price-dividend ratio is 20 .

Most importantly, the model produces a volatility smirk that is consistent with post-1987 market crash observation. Figure 1 reports implied volatilities for options on the S\&P 500 index with one month to maturity for the baseline case. The main result is that put options that are $10 \%$ out-ofthe-money have a $23.8 \%$ implied volatility, which is roughly consistent with the evidence in, e.g., 
Bakshi et al. (2003), Bates (2000), Eraker (2004), Foresi and Wu (2003), and Pan (2002). Further, at-the-money options have a $13.8 \%$ implied volatility. As such, the model predicts a realistic $10 \%$ volatility smirk, as measured by the difference in 10\%-out-of-the-money and at-the-money implied volatilities.

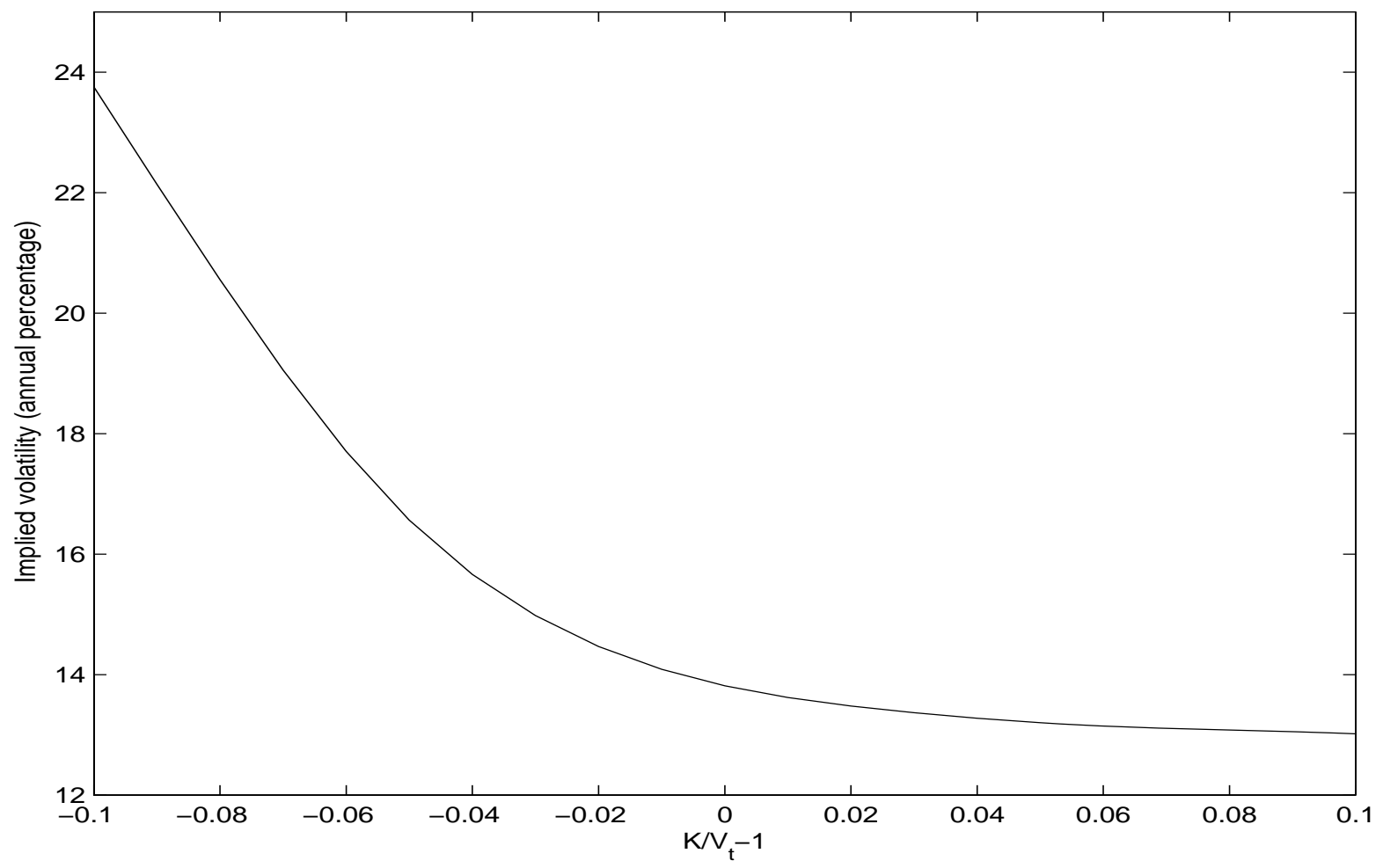

Figure 1: The plot depicts the implied volatility smirk for S\&P 500 options with one month to maturity. The model coefficients are set equal to the baseline values.

\subsection{Sensitivity Analysis}

Here we investigate the sensitivity of our findings to changes in the underlying parameters:

\section{Jump Coefficients}

Figure 2 illustrates the sensitivity of our results to the jump coefficients $\lambda$ and $\mu_{\nu}$. In the left panel we lower the jump intensity coefficient $\lambda$ to 0.01 , which corresponds to an expected arrival rate of one jump every 100 years. Interestingly, we find that most of the volatility smirk remains intact. As intuition would suggest, increasing the jump intensity to 0.03, i.e., one jump every 33 years, makes our results much stronger. 
In the right panel, we illustrate the effect of a one-standard-deviation perturbation of the average jump size coefficient. We note that in the model a value of $\mu_{\nu}=\left(-0.094+\sigma_{\nu}\right)=-0.079$ implies that a jump of average size determines a $20.6 \%$ fall in stock prices, which is smaller than the $24.5 \%$ drop in the S\&P 500 index observed in between the close of Thursday, October 15, and Monday, October 19, 1987. ${ }^{11}$ Still, the model predicts a steep volatility smirk.
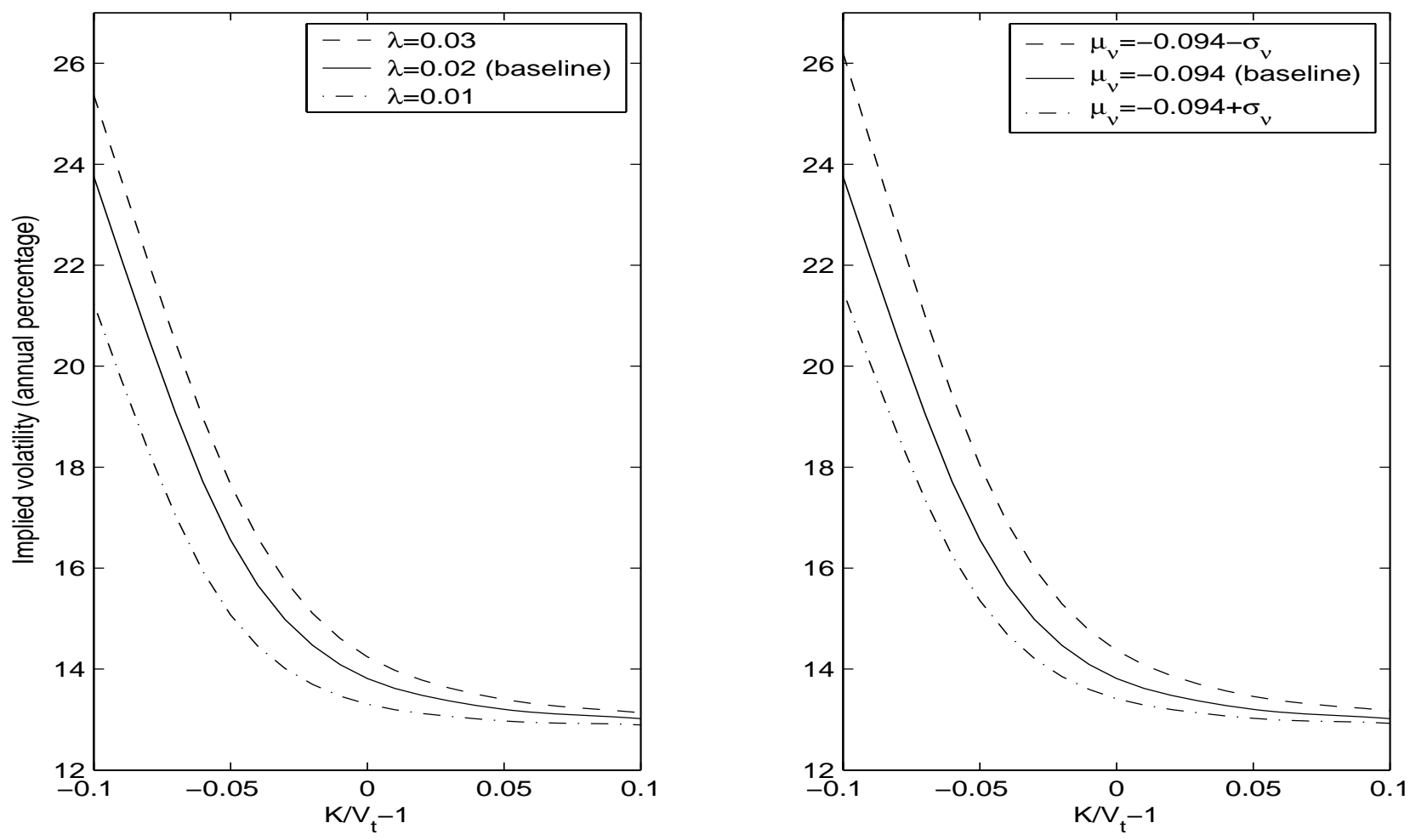

Figure 2: The plot illustrates the sensitivity of the implied volatility smirk to the agent's preferences coefficients, i.e., the jump intensity coefficient $\lambda$ and the average jump size coefficient $\mu_{\nu}$. Implied volatilities are from S\&P 500 options with one month to maturity.

\section{Preferences Coefficients}

Figure 3 illustrates the sensitivity of our results to the preferences coefficients $\gamma$ and $\Psi \equiv 1 / \rho$. The left panel shows that when the coefficient of risk aversion is lowered to 5 , most of the volatility smirk remains intact. Further, we note that when $\gamma=10$ (the upper bound of the range that Mehra and Prescott (1985) consider reasonable) the volatility smirk becomes considerably steeper.

\footnotetext{
${ }^{11}$ Note, however, that the drop in prices between the close of Friday October 16 and Monday October 19 was $20.46 \%$. Furthermore, the S\&P 500 closing prices over that week are as follows. 1987-10-13: \$314.52; 1987-10-14: $\$ 305.23$; 1987-10-15: $\$ 298.08 ; 1987-10-16$ : $\$ 282.94 ; 1987-10-19$ : $\$ 225.06 ; 1987-10-20$ : $\$ 236.84$.
} 
The right panel illustrates the sensitivity of the volatility smirk to the EIS coefficient. As noted previously, researchers have obtained a wide array of estimates for this parameter. Our base case estimate of $\rho=2$ is consistent with that of Bansal et al. (2004). Here we demonstrate that even lower estimates for $\rho$, such as 1.25 and 1.5 , still produce steep volatility smirks.
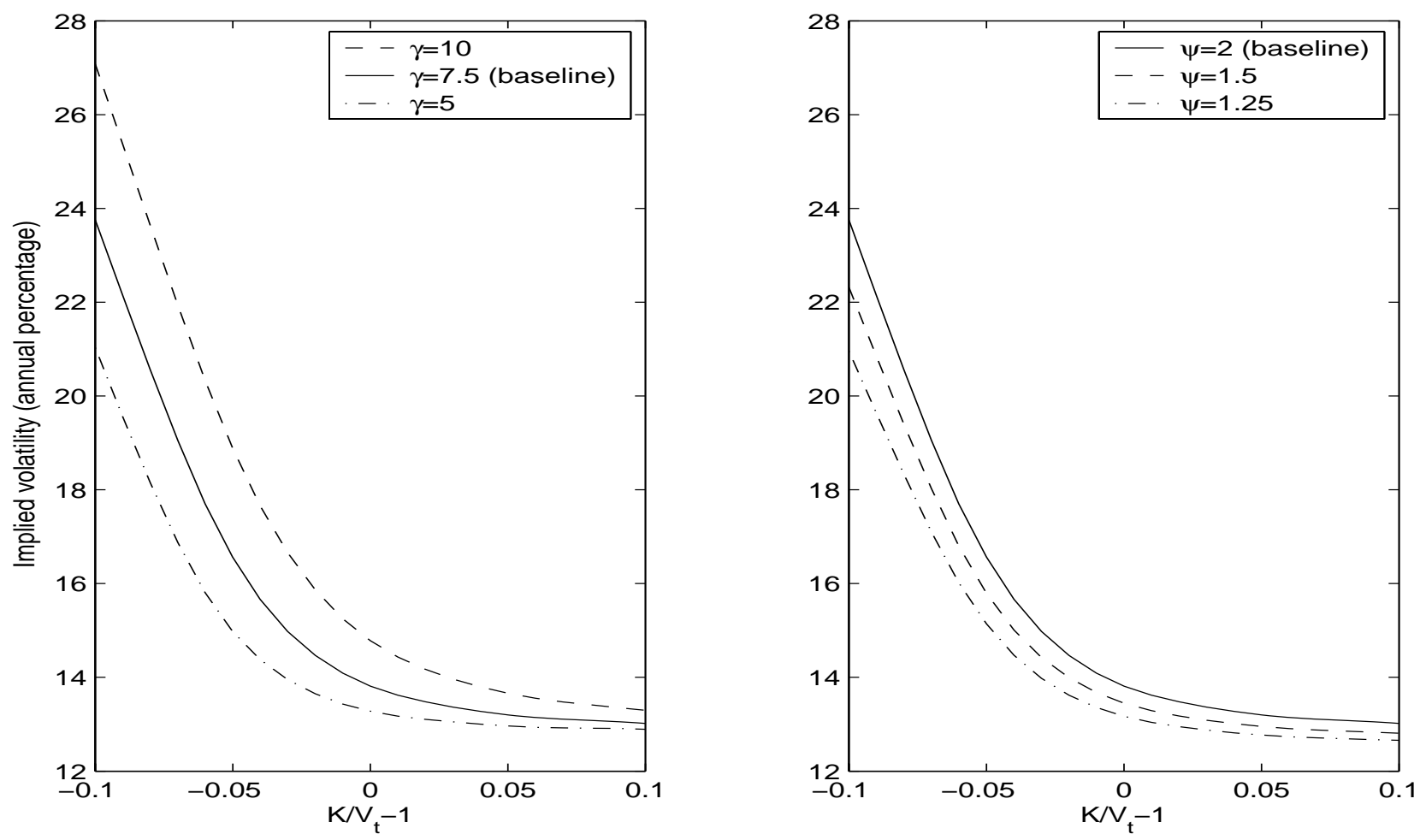

Figure 3: The plot illustrates the sensitivity of the implied volatility smirk to the agent's preferences coefficients, i.e., the coefficient of relative risk aversion $\gamma$ and the EIS $\Psi=\frac{1}{\rho}$. Implied volatilities are from S\&P 500 options with one month to maturity.

\section{Bayesian Updating of Jump Beliefs}

In this section, we examine whether our model can also explain the stark change in the implied volatility pattern that has maintained since the 1987 market crash. In the previous section, we assumed that the specified parameters of the model are known to the agent. In what follows, we will assume that, because stock market crashes are so rare, the agent does not know the exact distribution of the jump size. As such, she will update her prior beliefs about the distribution of jump size after observing a crash. Note that this Bayesian updating only occurs at crash dates. As such, the effect on the implied volatility pattern can be extremely long-lived. 
We specify the model so that, prior to the first crash, given the agent's information set, the distribution of the jump size $\widetilde{\nu}_{1}$ is a normal random variable whose mean value $\bar{\mu}_{\nu}$ is itself an unknown quantity, and is selected from a normal distribution:

$$
\begin{aligned}
\widetilde{\nu}_{1} \mid \bar{\mu}_{\nu} & \leadsto N\left(\bar{\mu}_{\nu}, \bar{\sigma}_{\nu}^{2}\right) \\
\bar{\mu}_{\nu} & \leadsto N\left(\overline{\bar{\mu}}_{\nu}, \overline{\bar{\sigma}}_{\nu}^{2}\right) .
\end{aligned}
$$

That is, before the first crash occurs, the agent's prior is

$$
\widetilde{\nu}_{1} \leadsto N\left(\overline{\bar{\mu}}_{\nu}, \bar{\sigma}_{\nu}^{2}+\overline{\bar{\sigma}}_{\nu}^{2}\right)
$$

After the first crash occurs and the agent observes the realization of $\widetilde{\nu_{1}}$, she updates her beliefs about the distribution of $\bar{\mu}_{\nu}$ via the projection theorem:

$$
\begin{aligned}
\mathrm{E}\left[\bar{\mu}_{\nu} \mid \widetilde{\nu}_{1}\right] & =\mathrm{E}\left[\bar{\mu}_{\nu}\right]+\frac{\operatorname{Cov}\left(\bar{\mu}_{\nu}, \widetilde{\nu}_{1}\right)}{\operatorname{Var}\left(\widetilde{\nu}_{1}\right)}\left(\widetilde{\nu}_{1}-\mathrm{E}\left[\widetilde{\nu}_{1}\right]\right) \\
& =\overline{\bar{\mu}}_{\nu}\left(\frac{\bar{\sigma}_{\nu}^{2}}{\bar{\sigma}_{\nu}^{2}+\overline{\bar{\sigma}}_{\nu}^{2}}\right)+\widetilde{\nu}_{1}\left(\frac{\overline{\bar{\sigma}}_{\nu}^{2}}{\bar{\sigma}_{\nu}^{2}+\overline{\bar{\sigma}}_{\nu}^{2}}\right) \\
\operatorname{Var}\left[\bar{\mu}_{\nu} \mid \widetilde{\nu}_{1}\right] & =\operatorname{Var}\left(\bar{\mu}_{\nu}\right)-\frac{\operatorname{Cov}\left(\bar{\mu}_{\nu}, \widetilde{\nu}_{1}\right)^{2}}{\operatorname{Var}\left(\widetilde{\nu}_{1}\right)} \\
& =\frac{\bar{\sigma}_{\nu}^{2} \overline{\bar{\sigma}}_{\nu}^{2}}{\bar{\sigma}_{\nu}^{2}+\overline{\bar{\sigma}}_{\nu}^{2}} .
\end{aligned}
$$

Hence, the agent sees the second crash size as distributed normally

$$
\widetilde{\nu}_{2} \leadsto N\left(\mathrm{E}\left[\bar{\mu}_{\nu} \mid \widetilde{\nu}_{1}\right], \bar{\sigma}_{\nu}^{2}+\operatorname{Var}\left[\bar{\mu}_{\nu} \mid \widetilde{\nu}_{1}\right]\right)
$$

We see from equation (43) that if the realization of $\widetilde{\nu}_{1}$ is substantially worse than the pre-crash estimate $\bar{\mu}_{\nu}$, then, after the first crash, the expected size of the next crash is considerably worse. Further, we emphasize that the random variable $\bar{\mu}_{\nu}$ is chosen only once at date- 0 , and hence uncertainty about its value is reduced at the crash date, as noted in equation (44). Indeed, prior to the crash the uncertainty about the value of $\bar{\mu}_{\nu}$ is $\overline{\bar{\sigma}}_{\nu}^{2}$, as can be seen from equation (41). However, after the crash, this uncertainty reduces to $\frac{\bar{\sigma}_{\nu}^{2} \overline{\bar{\sigma}}_{\nu}^{2}}{\bar{\sigma}_{\nu}^{2}+\overline{\bar{\sigma}}_{\nu}^{2}}=\frac{\bar{\sigma}_{\nu}^{2}}{1+\frac{\bar{\sigma}_{\nu}^{2}}{\overline{\bar{\sigma}}_{\nu}^{2}}}$. Below, we will parameterize the model so that $\overline{\bar{\sigma}}_{\nu}^{2} \ll \bar{\sigma}_{\nu}^{2}$. As such, most of the uncertainty regarding the value of $\bar{\mu}_{\nu}$ is determined from the first crash. While the agent would typically continue to update her beliefs about the distribution of $\bar{\mu}_{\nu}$ when subsequent crashes occur, given the parametrization of the model we choose below, there would be little change in the subsequent posterior beliefs. Therefore, and because it considerably simplifies the analysis, we make the assumption that the updating of jump beliefs occurs only once, 
when the agent observes a jump for the first time. Effectively this approach implies that the preand post-crash jump distributions are given by, respectively:

$$
\begin{aligned}
& \widetilde{\nu}_{1} \leadsto N\left(\overline{\bar{\mu}}_{\nu}, \bar{\sigma}_{\nu}^{2}+\overline{\bar{\sigma}}_{\nu}^{2}\right) \\
& \widetilde{\nu}_{j} \leadsto N\left\{\left[\overline{\bar{\mu}}_{\nu}\left(\frac{\bar{\sigma}_{\nu}^{2}}{\bar{\sigma}_{\nu}^{2}+\overline{\bar{\sigma}}_{\nu}^{2}}\right)+\widetilde{\nu}_{1}\left(\frac{\overline{\bar{\sigma}}_{\nu}^{2}}{\bar{\sigma}_{\nu}^{2}+\overline{\bar{\sigma}}_{\nu}^{2}}\right)\right], \bar{\sigma}_{\nu}^{2}+\frac{\bar{\sigma}_{\nu}^{2} \overline{\bar{\sigma}}_{\nu}^{2}}{\bar{\sigma}_{\nu}^{2}+\overline{\bar{\sigma}}_{\nu}^{2}}\right\} \quad j=2,3,, \ldots \infty .
\end{aligned}
$$

\subsection{Model Solution with Bayesian Updating}

We have assumed that the agent updates her beliefs only once, when she observes the first jump. As such, we only need to consider two cases when solving our problem. First, the case in which the agent is aware that stock market prices can jump, but she has not yet seen a jump occur. Second, the case in which the agent has witnessed a jump in market prices and therefore has updated her beliefs on the jump distribution. Intuitively, we can think of the first case as a description of the pre-1987 crash economy, while the second one depicts the post-1987 regime.

Once the agent has updated her beliefs in reaction to the occurrence of the first jump, the postcrash problem reduces to the setting without Bayesian updating that we have already considered in Sections 2.1-2.3. As such, the solution to the problem is unchanged, except that the mean $\mu_{\nu}$ and variance $\sigma_{\nu}^{2}$ in the jump distribution (5) are replaced by those of the post-crash jump distribution (47).

When solving the pre-crash problem, instead, we need to account for the fact that the agent rationally anticipates that the occurrence of a crash will determine an updating of the prior on the jump coefficients. To this end, we proceed as follows: As before, we exogenously specify the aggregate consumption and dividends dynamics as in equations (3)-(7). However, we now assume that the pre-crash jump size distribution is given by equation (46). Further, we consider a representative agent's whose preferences over the consumption process $\left\{C_{t}\right\}$ are represented by a utility index $U(t)$ that satisfies the recursive equation (8).

Proposition 1 in Appendix A still applies. As such, when $\rho, \gamma \neq 1$ the pre-crash value function $J_{\text {pre }}$ has the form:

$$
J_{p r e}=\frac{e^{c(1-\gamma)}}{1-\gamma} \beta^{\theta} I_{p r e}(x)^{\theta},
$$

where the price-consumption ratio $I_{\text {pre }}$ satisfies the following equation

$$
\begin{aligned}
0= & I_{p r e}\left((1-\gamma) \mu_{C}+(1-\gamma) x-\frac{\gamma}{2}(1-\gamma) \Omega-\beta \theta\right)-\kappa_{x} x \theta I_{p r e, x} \\
& +\frac{1}{2} \sigma_{x}^{2} \Omega \theta\left[(\theta-1)\left(\frac{I_{p r e, x}}{I_{p r e}}\right)^{2} I_{p r e}+I_{p r e, x x}\right]+\lambda I_{p r e} \mathrm{E}_{\widetilde{\nu}_{1}}\left[\frac{I_{p o s t}^{\theta}\left(x+\widetilde{\nu}_{1}\right)}{I_{p r e}^{\theta}(x)}-1\right]+\theta
\end{aligned}
$$


We note the effect of Bayesian updating on the pre-crash price-consumption ratio $I_{p r e}$. The agent anticipates that if a crash occurs, the price-consumption ratio will take the post-crash form

$$
I_{\text {post }}=e^{\tilde{A}+\tilde{B} x}
$$

where, for each different possible realization of $\widetilde{\nu}_{1}$, the coefficients $\tilde{A} \equiv A\left(\widetilde{\nu}_{1}\right)$ and $\tilde{B} \equiv B\left(\widetilde{\nu}_{1}\right)$ minimize the squared error in equation (17).

An approach similar to that followed in Section 2.1 delivers an approximate solution of the form

$$
I_{p r e}(x)=e^{A_{p r e}+B_{p r e} x}
$$

Specifically, we re-write equation (49) as

$$
\begin{aligned}
\left(p_{0}\right. & \left.+p_{1} x\right) e^{A_{p r e}+B_{p r e} x}-\theta-\lambda e^{(1-\theta)\left(A_{p r e}+B_{p r e} x\right)} \mathrm{E}_{\widetilde{\nu}_{1}}\left[e^{\theta\left(\tilde{A}+\tilde{B}\left(x+\widetilde{\nu}_{1}\right)\right)}\right]=\left(p_{0}+p_{1} x\right) e^{A_{p r e}+B_{p r e} x} \\
& +e^{A_{p r e}+B_{p r e} x}\left[(1-\gamma) \mu_{C}+(1-\gamma) x-\frac{\gamma}{2}(1-\gamma) \Omega-\beta \theta-\kappa_{x} x \theta B_{p r e}+\frac{1}{2} \sigma_{x}^{2} \Omega\left(\theta B_{p r e}\right)^{2}-\lambda\right] .(52)
\end{aligned}
$$

We set the RHS of (52) to zero and obtain a system of two equations, which identify the $\left\{p_{0}, p_{1}\right\}$ coefficients in terms of $B_{p r e}$ :

$$
\begin{aligned}
-p_{0} & =(1-\gamma) \mu_{C}-\frac{\gamma}{2}(1-\gamma) \Omega-\beta \theta+\frac{1}{2} \sigma_{x}^{2} \Omega\left(\theta B_{p r e}\right)^{2}-\lambda \\
-p_{1} & =(1-\gamma)-\kappa_{x} B_{p r e} \theta
\end{aligned}
$$

We then choose $\left\{A_{p r e}, B_{p r e}\right\}$ by minimizing the unconditional squared error:

$$
\min _{\left\{A_{p r e}, B_{p r e}\right\}} \mathrm{E}_{-\infty}\left\{\left(\left(p_{0}+p_{1} x\right) e^{A_{p r e}+B_{p r e} x}-\theta-\lambda e^{\left.(1-\theta)\left(A_{p r e}+B_{p r e} x\right)\right)} \mathrm{E}_{\widetilde{\nu}_{1}}\left[e^{\theta\left(\tilde{A}+\tilde{B}\left(x+\widetilde{\nu}_{1}\right)\right)}\right]\right)^{2}\right\} .
$$

Next, we derive the dynamics of the pre-crash pricing kernel:

$$
\begin{aligned}
\frac{d \Pi_{1}}{\Pi_{1}}= & -r_{p r e} d t-\gamma \sqrt{\Omega} d z_{C}+(\theta-1) B_{p r e} \sigma_{x} \sqrt{\Omega} d z_{x} \\
& +\left[\frac{e^{(\theta-1)\left(\tilde{A}+\tilde{B}\left(x+\widetilde{\nu}_{1}\right)\right)}}{e^{(\theta-1)\left(A_{p r e}+B_{p r e} x\right)}}-1\right] d N-\lambda \mathrm{E}_{\widetilde{\nu}_{1}}\left[\frac{e^{(\theta-1)\left(\tilde{A}+\tilde{B}\left(x+\widetilde{\nu}_{1}\right)\right)}}{e^{(\theta-1)\left(A_{p r e}+B_{p r e} x\right)}}-1\right] d t
\end{aligned}
$$

where the pre-crash risk-free rate $r_{p r e}$ is no longer an affine function of $x$ :

$$
\begin{aligned}
r_{p r e}= & r_{p r e, 0}+\rho x-\lambda \frac{(1-\theta)}{\theta} e^{-\theta\left(A_{p r e}+B_{p r e} x\right)} \mathrm{E}_{\widetilde{\nu}_{1}}\left[e^{\theta\left(\tilde{A}+\tilde{B}\left(x+\widetilde{\nu}_{1}\right)\right)}\right] \\
& -\lambda e^{(1-\theta)\left(A_{p r e}+B_{p r e} x\right)} \mathrm{E}_{\widetilde{\nu}_{1}}\left[e^{(\theta-1)\left(\tilde{A}+\tilde{B}\left(x+\widetilde{\nu}_{1}\right)\right)}\right] \\
r_{p r e, 0}= & \beta+\rho \mu_{C}-\frac{\gamma}{2} \Omega(1+\rho)-\frac{1}{2} \sigma_{x}^{2} \Omega(1-\theta) B_{p r e}^{2}+\frac{\lambda}{\theta} .
\end{aligned}
$$


Further, we obtain pre-crash risk-neutral dynamics:

$$
\begin{aligned}
d c & =\left(\mu_{C}+x-\Omega\left(\frac{1}{2}+\gamma\right)\right) d t+\sqrt{\Omega} d z_{C}^{Q} \\
d \delta & =\left(\mu_{D}+\phi x-\sigma_{D} \Omega\left(\frac{1}{2} \sigma_{D}+\rho_{C, D} \gamma\right)\right) d t+\sigma_{D} \sqrt{\Omega}\left(\rho_{C, D} d z_{C}^{Q}+\sqrt{1-\rho_{C, D}^{2}} d z_{D}^{Q}\right) \\
d x & =\left(-\kappa_{x} x-(1-\theta) B_{p r e} \sigma_{x}^{2} \Omega\right) d t+\sigma_{x} \sqrt{\Omega} d z_{x}^{Q}+\widetilde{\nu}_{1} d N,
\end{aligned}
$$

where the three Brownian motions $\left\{d z_{C}^{Q}, d z_{x}^{Q}, d z_{\Omega}^{Q}\right\}$ are uncorrelated, and the Q-intensity of the Poisson jump process $N$ is

$$
\lambda^{Q}=\lambda e^{(1-\theta)\left(A_{\text {pre }}+B_{\text {pre }} x\right)} \mathrm{E}_{\widetilde{\nu}_{1}}\left[e^{(\theta-1)\left(\tilde{A}+\tilde{B}\left(x+\widetilde{\nu}_{1}\right)\right)}\right] .
$$

Furthermore, the Q-probability density of the jump amplitudes is

$$
\pi^{Q}\left(\widetilde{\nu}_{1}=\nu_{1}\right)=\pi\left(\widetilde{\nu}_{1}=\nu_{1}\right) \frac{e^{(\theta-1)\left(A\left(\nu_{1}\right)+B\left(\nu_{1}\right)\left(x+\nu_{1}\right)\right.}}{\mathrm{E}_{\widetilde{\nu}_{1}}\left[e^{(\theta-1)\left(\tilde{A}+\tilde{B}\left(x+\widetilde{\nu}_{1}\right)\right)}\right]}
$$

\subsection{Pre-Crash Dividend Claim}

We denote the pre-crash claim to dividend by $V_{p r e}(D, x)$. By construction, its expected return under the risk neutral measure is the risk-free rate:

$$
\mathrm{E}_{t}^{Q}\left[\frac{d V_{p r e}+D d t}{V_{p r e}}\right]=r_{p r e} d t .
$$

We proceed as in Section 2.3. That is, we define the pre-crash price-dividend ratio $I_{p r e}^{D} \equiv \frac{V_{p r e}}{D}$ and then look for a solution of the form

$$
I_{p r e}^{D}(x)=e^{F_{p r e}+G_{p r e} x}
$$

We combine equations (63)-(64) with the risk-neutral dynamics (59)-(60) to obtain:

$$
\begin{aligned}
r_{p r e}-\frac{1}{I_{p r e}^{D}}= & \mu_{D}+\phi x-\gamma \rho_{C, D} \sigma_{D} \Omega-\kappa_{x} x G_{p r e}-(1-\theta) B_{p r e} G_{p r e} \sigma_{x}^{2} \Omega+\frac{1}{2} G_{p r e}^{2} \sigma_{x}^{2} \Omega \\
& +\lambda^{Q} e^{-\left(F_{p r e}+G_{p r e} x\right)} \mathrm{E}_{\widetilde{\nu}_{1}}^{Q}\left[e^{\tilde{F}+\tilde{G}\left(x+\widetilde{\nu}_{1}\right)}\right]-\lambda^{Q},
\end{aligned}
$$

As above, we find an approximate solution for $I_{\text {pre }}^{D}$ by moving $r_{p r e}$ to the RHS, arranging the nonaffine terms to the LHS, multiplying both sides by $I_{\text {pre }}^{D}$, and adding $\left(q_{0}+q_{1} x\right) I_{\text {pre }}^{D}$ to both sides. These calculations give

$$
L H S=\left(q_{0}+q_{1} x\right) e^{F_{p r e}+G_{p r e} x}-1-\lambda \frac{(1-\theta)}{\theta} e^{\left(F_{p r e}+G_{p r e} x\right)-\theta\left(A_{p r e}+B_{p r e} x\right)} \mathrm{E}_{\widetilde{\nu}_{1}}\left[e^{\theta\left(\tilde{A}+\tilde{B}\left(x+\widetilde{\nu}_{1}\right)\right)}\right]
$$




$$
\begin{aligned}
& -\lambda e^{\left(F_{p r e}+G_{p r e} x\right)+(1-\theta)\left(A_{p r e}+B_{p r e} x\right)} \mathrm{E}_{\widetilde{\nu}_{1}}\left[e^{(\theta-1)\left(\tilde{A}+\tilde{B}\left(x+\widetilde{\nu}_{1}\right)\right)}\right]-\lambda^{Q} \mathrm{E}_{\widetilde{\nu}_{1}}^{Q}\left[e^{\tilde{F}+\tilde{G}\left(x+\widetilde{\nu}_{1}\right)}\right] \\
\left(\frac{1}{I_{p r e}^{D}}\right) R H S= & \left(q_{0}+q_{1} x\right)-r_{p r e, 0}+\mu_{D}-\sigma_{D} \rho_{C, D} \gamma \Omega-(1-\theta) B_{p r e} G_{p r e} \sigma_{x}^{2} \Omega+\frac{1}{2} G_{p r e}^{2} \sigma_{x}^{2} \Omega-\lambda^{Q} \\
& +\phi x-\kappa_{x} x G_{p r e}-\rho x
\end{aligned}
$$

where the constant $r_{p r e, 0}$ is defined in equation (57). We note the effect of Bayesian updating on the pre-crash price-dividend ratio $I_{p r e}^{D}$. The agent anticipates that if a crash occurs the price-dividend ratio will take the post-crash form

$$
I_{\text {post }}^{D}=e^{\tilde{F}+\tilde{G} x}
$$

where, for each possible realization of $\widetilde{\nu}_{1}$, the coefficients $\tilde{F} \equiv F\left(\widetilde{\nu}_{1}\right)$ and $\tilde{G} \equiv G\left(\widetilde{\nu}_{1}\right)$ minimize the squared error in equation (37).

We approximate the RHS to be identically zero, and then collect terms linear in and independent of $x$, respectively. We obtain a system of two equations that identify $\left\{q_{0}, q_{1}\right\}$ in terms of $G_{p r e}$ :

$$
\begin{aligned}
-q_{0} & =-r_{p r e, 0}+\mu_{D}-\sigma_{D} \rho_{C, D} \gamma \Omega-(1-\theta) B_{p r e} G_{p r e} \sigma_{x}^{2} \Omega+\frac{1}{2} G_{p r e}^{2} \sigma_{x}^{2} \Omega-\lambda^{Q} \\
-q_{1} & =\phi-\kappa_{x} G_{p r e}-\rho .
\end{aligned}
$$

In turn, we identify $\left\{F_{p r e}, G_{p r e}\right\}$ by minimizing the unconditional squared error:

$$
\min _{\left\{F_{p r e}, G_{p r e}\right\}} \mathrm{E}_{-\infty}\left[(L H S)^{2}\right] .
$$

\subsection{The Pre-Crash Equity Premium}

In the pre-crash economy, the expression for the risk premium on the risky asset simplifies to:

$$
\begin{aligned}
\text { Equity Premium }_{\text {pre }} & =\gamma \sigma_{D} \rho_{C, D} \Omega+(1-\theta) B_{p r e} G_{p r e} \sigma_{x}^{2} \Omega \\
& -\lambda I_{p r e}^{(1-\theta)}\left(I_{p r e}^{D}\right)^{-1} \mathrm{E}_{\widetilde{\nu}_{1}}\left[e^{(\theta-1)\left(\tilde{A}+\tilde{B}\left(x+\widetilde{\nu}_{1}\right)\right)+\tilde{F}+\tilde{G}\left(x+\widetilde{\nu}_{1}\right)}\right] \\
& +\lambda I_{p r e}^{(1-\theta)} \mathrm{E}_{\widetilde{\nu}_{1}}\left[e^{(\theta-1)\left(\tilde{A}+\tilde{B}\left(x+\widetilde{\nu}_{1}\right)\right)}\right]+\lambda\left(I_{p r e}^{D}\right)^{-1} \mathrm{E}_{\widetilde{\nu}_{1}}\left[e^{\tilde{F}+\tilde{G}\left(x+\widetilde{\nu}_{1}\right)}\right]-\lambda
\end{aligned}
$$

where $I_{p r e}$ and $I_{\text {pre }}^{D}$ were previously defined in equations (51) and (64), respectively.

The intuition for this formula is similar to that discussed previously in Section 2.4. That is, the first term in equation (72) is identical to the risk premium in a model with CRRA. The following terms are the risk premia on diffusive and jump components of expected growth risk. Again, in the KPEZ with $\Psi>1$ case, the agent demands a positive premium on expected growth risk, which increases the risk premium on the risky asset. 


\subsection{Valuing Options on the Dividend Claim}

The option pricing problem for the pre-crash economy is outside of the affine class. Thus, we lack an analytical formula for the option price. However, the problem is easily handled via Monte Carlo simulation. Specifically, we simulate two antithetic samples of 50,000 paths of the dividend $\delta$ and the process $x$ from the Q-dynamics (23) and (24). For each simulated case, we use the

$x$-path from time $t$ to maturity $T$ to approximate the discount factor $e^{-\int_{t}^{T} r\left(x_{s}\right) d s}$. Further, we use the simulated value of $x_{T}$ to obtain the price-dividend ratio $I_{\text {pre }}^{D}(T)=e^{F_{p r e}+G_{p r e} x_{T}}$. Next, we compute the simulated value of the contingent claim $V_{p r e}(T)=D_{T} I_{p r e}^{D}(T)$, where $D=\exp \delta$. Finally, we average across the simulated discounted realizations of $\left|V_{p r e}(T)-K\right|^{+}$to approximate the expectation in (39).

\subsection{Model Calibration}

We note that the requirements imposed on this model is considerably higher than in the previous section in that here we want to explain not only the post-1987 volatility smirk, but also the regime shift in option prices that was observed immediately after the 1987 crash. As such, we consider a slightly different baseline calibration. We argue that the coefficient values that we use below are still consistent with observation and similar to those used in, e.g., BY and Bansal et al. (2004).

\section{Consumption and Dividend Dynamics:}

In the consumption dynamics (6), we fix $\mu_{C}=0.018$ and $\Omega=0.00078$.

For the dividend process (7), we use $\mu_{D}=0.018, \phi=2.1$, and $\sigma_{D}=3.5$. We fix the correlation between shocks to dividend and consumption at $25 \%$, i.e., $\rho_{C, D}=0.25$.

In the $x$-dynamics (3), we use $\kappa_{x}=0.34$ and $\sigma_{x}=0.6325$. We fix the Poisson jump intensity process at $\lambda=0.007$, which on average corresponds to less than one jump every hundred years.

In equations (46)-(47), we fix $\overline{\bar{\mu}}_{\nu}=-0.011$ and we assume that at the time of the crash $\widetilde{\nu}_{1}$ takes value -0.094 . Further, we set $\bar{\sigma}_{\nu}=0.0023$ and $\overline{\bar{\sigma}}_{\nu}=0.022$.

The intuition for this calibration is as follows. Before a crash occurs, the agent does not fully appreciate the extent to which prices can fall. As such, her prior is that the jump size $\widetilde{\nu}_{1}$ has nearly zero mean, $\overline{\bar{\mu}}_{\nu}=-0.011$. The agent realizes however that there is considerable uncertainty about the magnitude of a possible jump, as reflected by the large standard deviation of $\widetilde{\nu}_{1}$, which equals $\sqrt{\bar{\sigma}_{\nu}^{2}+\overline{\bar{\sigma}}_{\nu}^{2}}=0.0221$. 
Suddenly, she unexpectedly observes a crash of the proportion of the 1987 event. When that happens, she updates her beliefs about the post-crash jump distribution according to (47). As such, the mean and standard deviation of the post-crash jump size $\widetilde{\nu}_{2}$ become, respectively,

$$
\begin{aligned}
\overline{\bar{\mu}}_{\nu}\left(\frac{\bar{\sigma}_{\nu}^{2}}{\bar{\sigma}_{\nu}^{2}+\overline{\bar{\sigma}}_{\nu}^{2}}\right)+\widetilde{\nu}_{1}\left(\frac{\overline{\bar{\sigma}}_{\nu}^{2}}{\bar{\sigma}_{\nu}^{2}+\overline{\bar{\sigma}}_{\nu}^{2}}\right) & =-0.0931 \\
\sqrt{\bar{\sigma}_{\nu}^{2}+\frac{\bar{\sigma}_{\nu}^{2} \overline{\bar{\sigma}}_{\nu}^{2}}{\overline{\bar{\sigma}}_{\nu}^{2}+\overline{\bar{\sigma}}_{\nu}^{2}}} & =0.0032 .
\end{aligned}
$$

That is, immediately after the crash the agent updates her prior on the average jump size in a way that reflects the possibility of a large, although very rare, stock price fall.

Further, we note that the occurrence of a crash determines a stark increase in the precision of the agent's belief about the jump size. Specifically, the standard deviation of the postcrash jump size is over seven times smaller than its pre-crash value. As discussed above, this observation is consistent with the intuition that a single event of the proportion of the 1987 market crash can generate most of the updating of the agent's beliefs.

\section{Preferences:}

We use a time discount factor coefficient $\beta=0.017$. We fix the coefficient of relative risk aversion at $\gamma=10$. Finally, we follow Bansal et al. (2004) and we use $\Psi=1 / \rho=2$ for our baseline case.

\section{Initial Conditions:}

In the plots below, we fix the state variable $x$ at the its steady-state mean value. In the pre-crash economy, such value is $x_{\text {pre }, 0}=\overline{\bar{\mu}}_{\nu} \lambda / \kappa_{x}$, while in the post-crash economy it is

$x_{\text {post }, 0}=\left[\overline{\bar{\mu}}_{\nu}\left(\frac{\bar{\sigma}_{\nu}^{2}}{\overline{\bar{\sigma}}_{\nu}^{2}+\overline{\bar{\sigma}}_{\nu}^{2}}\right)+\widetilde{\nu}_{1}\left(\frac{\overline{\bar{\sigma}}_{\nu}^{2}}{\overline{\bar{\sigma}}_{\nu}^{2}+\overline{\bar{\sigma}}_{\nu}^{2}}\right)\right] \lambda / \kappa_{x}$. We also confirmed, however, that our results are robust to the choice of a wide range of values for the state $x$.

\subsection{Simulation Results}

We note that our calibration yields realistic values of the risk-free rate and the equity premium, both pre- and post-crash. Specifically, we find that the pre-crash steady-state real risk-free rate is $1.33 \%$, while the equity premium predicted by the model is $4.48 \%$. Post-crash, the steady-state value of the risk-free rate drops to $0.7 \%$, while the equity premium becomes $6.4 \%$. Further, the calibration matches other aspects of the economy. For instance, we find that the steady-state value 
of the price-dividend ratio is around 27, a value that drops to approximately 19 in the post-crash economy.

Rubinstein (1994) argues that until 1987 the Black-Scholes formula worked quite well to explain S\&P 500 index option prices. Similarly, Bates (2000) notes that pre-crash implied volatilities from out-of-the-money puts on the S\&P 500 futures where almost invariably higher than those from at-the-money options. However, pre-crash implicit volatilities from out-of-the-money calls were sometimes below and sometimes above those from at-the-money options. As such, pre-crash implied volatilities displayed either a mild 'smile' or tenuous 'smirk' pattern. Our calibration produces a mild smirk that is qualitatively consistent with the Bates evidence. As we illustrate in Figure 4, the difference between the implied volatilities from 10\%-out-of-the-money and at-the-money puts is approximately $3 \%$.

Immediately after the crash, however, the agent updates her beliefs about the expected value of the jump size. As such, the volatility smirk steepens dramatically. In Figure 4, we show that post1987 the difference between the implied volatilities from 10\%-out-of-the-money and at-the-money puts becomes nearly $10 \%$.

Finally, we note a drawback of our calibration. During the two weeks after the 'Black Monday' in October 1987, the 3-month Treasury bill rate was on average 1.5\% lower than the same rate during the two weeks preceeding the crash. ${ }^{12}$ Consistent with observation, our model predicts a fall in the risk-free rate at the time of a market crash. However, the magnitude of the drop is larger than what was observed in October 1987. To study this model implication, we use data from the COMPUSTAT database and compute the price-dividend ratio for the S\&P 500 index as of the end of September 1987. We find it to be 36.24. Next, we infer the pre-crash value $x_{t}$ of the latent process $x$ (where $t$ is the end of September 1987) by matching the pre-crash price-dividend ratio predicted by the model with the value observed in the data:

$$
I_{\text {pre }}^{D}\left(x_{t}\right)=e^{F_{p r e}+G_{p r e} x_{t}} \equiv 36.24 .
$$

Then, we use equation (57) to compute the change in the risk-free rate determined by a jump in $x$ from the pre-crash value $x_{t}$ to the post-crash level $x_{t}+\widetilde{\nu}_{1}$. We find the jump in the risk-free rate to be $-5.2 \%$.

Related, we can use our model to predict the drop in stock prices at the time of the 1987 crash. We do so by following an approach similar to that we used above to determine the jump in the

\footnotetext{
${ }^{12}$ The bank discount rates on the 3M T-bill were as follows. 1987-10-05: 6.68; 1987-10-06: 6.55; 1987-10-07: 6.56; 1987-10-08: 6.69; 1987-10-09: 6.75; 1987-10-12: N.A.; 1987-10-13: 6.74; 1987-10-14: 7.19; 1987-10-15: 7.07; 1987-1016: 6.93; 1987-10-19: 6.39; 1987-10-20: 5.86; 1987-10-21: 5.60; 1987-10-22: 5.36; 1987-10-23: 5.29; 1987-10-26: 5.22; 1987-10-27: 5.23; 1987-10-28: 5.10; 1987-10-29: 5.03; 1987-10-30: 5.27.
} 


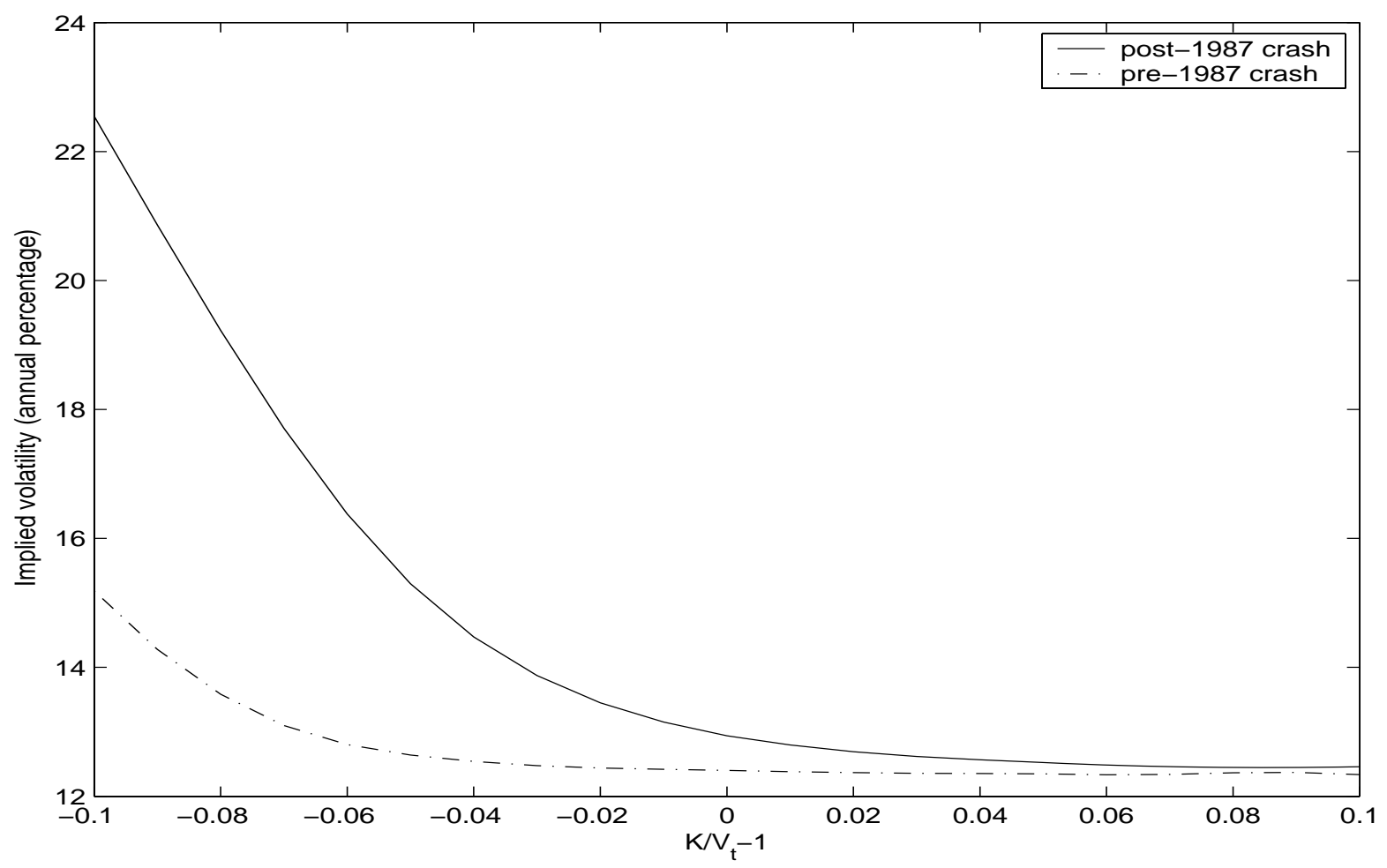

Figure 4: The plot depicts the implied volatility smirk pre- and post-1987 market crash. Implied volatilities are from S\&P 500 options with one month to maturity. The model coefficients are set equal to the baseline values.

interest rate. That is, assuming that the level of the dividend is unaffected by the crash, the jump in price around the crash event is given by

$$
\frac{I^{D}\left(x_{t}+\widetilde{\nu}_{1}\right)}{I_{p r e}^{D}\left(x_{t}\right)}-1=\frac{e^{F+G\left(x_{t}+\widetilde{\nu}_{1}\right)}}{e^{F_{p r e}+G_{p r e} x_{t}}}-1,
$$

where $x_{t}$ is determined by equation (75) and $\widetilde{\nu}_{1}$ is the jump in $x$ at the time of the crash. The model predicts a nearly fifty percent fall in the stock price, a drop twice as large as that observed in 1987.

\section{Conclusions}

Prior to the stock market crash of 1987, the Black-Scholes model of option prices worked rather well across all strikes. Since the crash, however, deep out-of-the-money S\&P 500 put options have become 'expensive' relative to the Black-Scholes benchmark. These observations motivate two important questions. First, whether there exists a reasonable model for the endowment process 
and the preferences of agents that can explain the post-1987 SPX prices. Second, whether such a model can also explain the stark change in the volatility smirk that has maintained since the 1987 market crash. These are the questions that we address in the paper.

Many researchers (e.g., Liu, Pan and Wang (2005)) have investigated the first of the two questions. In general, they conclude that the prices of these securities cannot be justified in a general equilibrium setting if the representative agent is endowed with 'standard preferences'. In this paper, however, we demonstrate that the prices of these securities can be reconciled if the agent is endowed with Epstein-Zin preferences and if the expected growth rate of the aggregate dividend can experience (rare) jumps. We identify a realistic calibration of the model that simultaneously matches the empirical properties of dividends, the equity premium, and the level of the risk-free rate. Most importantly, we find that the agent, concerned with the possibility of a market crash, is willing to pay a high premium to buy out-of-the-money put options and hedge downside risk. Specifically, in our baseline calibration the implied volatility of $10 \%$ out-of-the-money put options with one month to maturity is close to $24 \%$. At-the-money options, instead, have an implied volatility of approximately $14 \%$. That is, consistent with empirical evidence we can generate a $10 \%$ volatility smirk.

We then proceed to investigate the second question, namely, whether the model can also explain the regime change observed in S\&P 500 prices immediately after the 1987 market crash. We note that an extreme event such as the 1987 market crash is likely to dramatically change the investor's perception about the nature of possible future market fluctuations. To formalize this intuition, we extend the model to a Bayesian setting in which the agent uses the information on a jump in market prices to update her belief about the expected magnitude of future jumps. Specifically, we assume that the jump size is a normal random variable whose mean value is itself a normally distributed random variable. The agent formulates a prior on the mean value of the jump size, and updates her prior when she observes an extreme event such as the 1987 crash.

We find that the model can explain the time variation in the shape of the volatility smirk. Specifically, we present simulation results in which the steepness of the pre-crash volatility smirk (i.e., the difference between implied volatilities from 10\%-out-of-the-money and at-the-money puts) is approximately $3 \%$, a number that is consistent with the pre-crash evidence reported in, e.g., Bates (2000). At the same time, the occurrence of a jump triggers the updating of the agent's beliefs about the expected value of the jump size. As such, after the crash out-of-the-money put options are perceived to be more valuable, and the volatility smirk becomes as steep as $10 \%$, consistent with the post-crash evidence. 
Our model shows that a single channel (a rare jump in consumption growth) suffices to reconcile option and index prices. However, for the case with Bayesian updating, the model consistent with pre- and post-crash data seems to predict a crash on the day of the event larger than what was observed in 1987. We note that there are several channels available that would most likely improve the fit on this dimension also. For instance, as in Collin Dufresne et al. (2003), we can allow for Bayesian updating not only on the size of the jump, but also on its intensity, i.e., on the probability that a jump will occur. Further, following BY we could model volatility as stochastic (possibly with jumps). We leave these interesting extensions to future research.

\section{Appendix A: Equilibrium Prices in a Jump-Diffusion Exchange Economy with Recursive Utility}

There are several formal treatments of stochastic differential utility and its implications for asset pricing (see, e.g., Duffie and Epstein (1992a,b), Duffie and Skiadas (1994), Schroder and Skiadas (1999, 2003), and Skiadas (2003)). For completeness, in this Appendix we offer a very simple informal derivation of the pricing kernel that obtains in an exchange economy where the representative agent has a KPEZ recursive utility. Our contribution is to characterize equilibrium prices in an exchange economy where aggregate output has particular jump-diffusion dynamics (Propositions 1 and 2).

\subsection{Representation of Preferences and Pricing Kernel}

We assume the existence of a standard filtered probability space $\left(\Omega, \mathcal{F},\left\{\mathcal{F}_{t}\right\}, P\right)$ on which there exists a vector $z(t)$ of $d$ independent Brownian motions and one counting process $N(t)=\sum_{i} \mathbf{1}_{\left\{\tau_{i} \leq t\right\}}$ for a sequence of inaccessible stopping times $\tau_{i}, i=1,2, \ldots{ }^{13}$

Aggregate consumption in the economy is assumed to follow a continuous process, with stochastic growth rate and volatility, which both may experience jumps:

$$
\begin{aligned}
d \log C_{t} & =\mu_{C}\left(X_{t}\right) d t+\sigma_{C}\left(X_{t}\right) d z(t) \\
d X_{t} & =\mu_{x}\left(X_{t}\right) d t+\sigma_{x}\left(X_{t}\right) d z(t)+\widetilde{\nu} d N(t) .
\end{aligned}
$$

We note that $X_{t}$ is a $n$-dimensional Markov process (we assume sufficient regularity on the coefficient of the stochastic differential equation (SDE) for it to be well-defined, e.g., Duffie (2001) Appendix B). In particular $\mu_{x}$ is an $(n, 1)$ vector, $\sigma_{x}$ is an $(n, d)$ matrix and $\widetilde{\nu}$ is a $(n, 1)$ vector of i.i.d. random

\footnotetext{
${ }^{13}$ We note that $N(t)$ is a pure jump process by construction and hence is independent of $z(t)$ by construction (in the sense that their quadratic co-variation is zero).
} 
variable with joint density (conditional on a jump $d N(t)=1$ ) of $g(\nu)$. We further assume that the counting process has a (positive integrable) intensity $\lambda\left(X_{t}\right)$ in the sense that $N(t)-\int_{0}^{t} \lambda\left(X_{s}\right) d s$ is a $\left(P, \mathcal{F}_{t}\right)$ martingale.

Following Epstein and Zin (1989), we assume that the representative agent's preferences over a consumption process $\left\{C_{t}\right\}$ are represented by a utility index $U(t)$ that satisfies the following recursive equation:

$$
U(t)=\left\{\left(1-e^{-\beta d t}\right) C_{t}^{1-\rho}+e^{-\beta d t} \mathrm{E}_{t}\left(U(t+d t)^{1-\gamma}\right)^{\frac{1-\rho}{1-\gamma}}\right\}^{\frac{1}{1-\rho}}
$$

With $d t=1$, this is the discrete time formulation of KPEZ, in which $\Psi \equiv 1 / \rho$ is the EIS and $\gamma$ is the risk-aversion coefficient.

To simplify the derivation let us define the function

$$
u_{\alpha}(x)=\left\{\begin{array}{cc}
\frac{x^{1-\alpha}}{(1-\alpha)} & 0<\alpha \neq 1 \\
\log (x) & \alpha=1
\end{array}\right.
$$

Further, let us define

$$
g(x)=u_{\rho}\left(u_{\gamma}^{-1}(x)\right) \equiv\left\{\begin{array}{cc}
\frac{((1-\gamma) x)^{1 / \theta}}{(1-\rho)} & \gamma, \rho \neq 1 \\
u_{\rho}\left(e^{x}\right) & \gamma=1, \rho \neq 1 \\
\frac{\log ((1-\gamma) x)}{(1-\gamma)} & \rho=1, \gamma \neq 1
\end{array}\right.
$$

where

$$
\theta=\frac{1-\gamma}{1-\rho}
$$

Then defining the 'normalized' utility index $J$ as the increasing transformation of the initial utility index $J(t)=u_{\gamma}(U(t))$ equation (79) becomes simply:

$$
g(J(t))=\left(1-e^{-\beta d t}\right) u_{\rho}\left(C_{t}\right)+e^{-\beta d t} g\left(\mathrm{E}_{t}[J(t+d t)]\right)
$$

Using the identity $J(t+d t)=J(t)+d J(t)$ and performing a simple Taylor expansion we obtain:

$$
0=\beta u_{\rho}\left(C_{t}\right) d t-\beta g(J(t))+g^{\prime}(J(t)) \mathrm{E}_{t}[d J(t)]
$$

Slightly rearranging the above equation, we obtain a backward recursive stochastic differential equation which could be the basis for a formal definition of stochastic differential utility (see Duffie Epstein (1992), Skiadas (2003)):

$$
\mathrm{E}_{t}[d J(t)]=-\frac{\beta u_{\rho}\left(C_{t}\right)-\beta g(J(t))}{g^{\prime}(J(t))} d t .
$$


Indeed, let us define the so-called 'normalized' aggregator function:

$$
f(C, J)=\frac{\beta u_{\rho}(C)-\beta g(J)}{g^{\prime}(J)} \equiv\left\{\begin{array}{cc}
\frac{\beta u_{\rho}(C)}{((1-\gamma) J)^{1 / \theta-1}}-\beta \theta J & \gamma, \rho \neq 1 \\
(1-\gamma) \beta J \log (C)-\beta J \log ((1-\gamma) J) & \gamma \neq 1, \rho=1 \\
\frac{\beta u_{\rho}(C)}{e^{(1-\rho) J}}-\frac{\beta}{1-\rho} & \gamma=1, \rho \neq 1 .
\end{array}\right.
$$

We obtain the following representation for the normalized utility index:

$$
J(t)=\mathrm{E}_{t}\left(\int_{t}^{T} f\left(C_{s}, J(s)\right)+J(T)\right) .
$$

Further, if the transversality condition $\lim _{T \rightarrow \infty} \mathrm{E}_{t}(J(T))=0$ holds, letting $T$ tend to infinity, we obtain the simple representation:

$$
J(t)=\mathrm{E}_{t}\left(\int_{t}^{\infty} f\left(C_{s}, J(s)\right) d s\right) .
$$

Fisher and Gilles (1999) discuss many alternative representations and choices of the utility index and associated aggregator as well as their interpretations. Here we note only the well-known fact that when $\rho=\gamma$ (i.e., $\theta=1$ ) then $f(C, J)=\beta u_{\rho}(C)-\beta J$ and a simple application of Itô's lemma shows that

$$
J(t)=\mathrm{E}_{t}\left(\int_{t}^{\infty} e^{-\beta(s-t)} \beta u_{\rho}\left(C_{s}\right) d s\right) .
$$

To obtain an expression for the pricing kernel note that under the assumption (which we maintain throughout) that an 'interior' solution to the optimal consumption-portfolio choice of the agent exists, a necessary condition for optimality is that the gradient of the Utility index is zero for any small deviation of the optimal consumption process in a direction that is budget feasible. More precisely, let us define the utility index corresponding to such a small deviation by:

$$
J^{\delta}(t)=\mathrm{E}_{t}\left(\int_{t}^{\infty} f\left(C_{s}^{*}+\delta \tilde{C}(s), J^{\delta}(s)\right) d s\right)
$$

Then we may define the gradient of the utility index evaluated at the optimal consumption process $C^{*}(t)$ in the direction $\tilde{C}(t)$ :

$$
\begin{aligned}
\nabla J\left(C_{t}^{*} ; \tilde{C}_{t}\right) & =\lim _{\delta \rightarrow 0} \frac{J^{\delta}(t)-J(t)}{\delta} \\
& =\lim _{\delta \rightarrow 0} \mathrm{E}_{t}\left[\int_{t}^{\infty} \frac{f\left(C_{s}^{*}+\delta \tilde{C}(s), J^{\delta}(s)\right)-f\left(C_{s}, J^{\delta}(s)\right)}{\delta} d s\right] \\
& =\mathrm{E}_{t}\left[\int_{t}^{\infty} f_{C}\left(C_{s}^{*}, J(s)\right) \tilde{C}_{s}+f_{J}\left(C_{s}, J(s)\right) \nabla J\left(C_{s}^{*} ; \tilde{C}_{s}\right) d s\right]
\end{aligned}
$$


Assuming sufficient regularity (essentially the gradient has to be a semi-martingale and the transver-

sality condition has to hold: $\lim _{T \rightarrow \infty} \mathrm{E}_{t}\left[e^{\int_{t}^{T} f_{J}\left(C_{s}, J_{s}\right) d s} \nabla J\left(C_{T}^{*} ; \tilde{C}_{T}\right)=0\right)$, a simple application of the generalized Itô-Doeblin formula gives the following representation:

$$
\nabla^{\delta} J\left(C_{t}^{*} ; \tilde{C}_{t}\right)=\mathrm{E}_{t}\left(\int_{t}^{\infty} e^{\int_{t}^{s} f_{J}\left(C_{u}, J_{u}\right) d u} f_{C}\left(C_{s}, J_{s}\right) \tilde{C}_{s} d s\right)
$$

This shows that

$$
\Pi(t)=e^{\int_{0}^{t} f_{J}\left(C_{s}, J_{s}\right) d s} f_{C}\left(C_{t}, J_{t}\right)
$$

is the Riesz representation of the gradient of the normalized utility index at the optimal consumption. Since a necessary condition for optimality is that $\nabla J\left(C_{t}^{*} ; \tilde{C}_{t}\right)=0$ for any feasible deviation $\tilde{C}_{t}$ from the optimal consumption stream $C_{t}^{*}$, we conclude that $\Pi(t)$ is a pricing kernel for this economy. ${ }^{14}$

\subsection{Equilibrium Prices}

Assuming equilibrium consumption process given in (77)-(78) above we obtain an explicit characterization of the felicity index $J$ and corresponding pricing kernel $\Pi$.

For this we define the operator for any $h(\cdot): \mathbb{R}^{n}-\mathbb{R}$ :

$$
\mathcal{J} h(x)=\int \ldots \int \frac{h(x+\nu)}{h(x)} g(\nu) d \nu_{1} \ldots d \nu_{n}-1
$$

and the standard Dynkin operator:

$$
\mathcal{D} h(x)=h_{x}(x) \mu_{x}(x)+\frac{1}{2} \operatorname{trace}\left(h_{x x} \sigma_{x}(x) \sigma_{x}(x)^{\top}\right),
$$

where $h_{x}$ is the $(n, 1)$ Jacobian vector of first derivatives and $h_{x x}$ denotes the $(n, n)$ Hessian matrix of second derivatives. with these notations, we find:

Proposition 1 Suppose $I(x): \mathbb{R}^{n} \rightarrow \mathbb{R}$ solves the following equation:

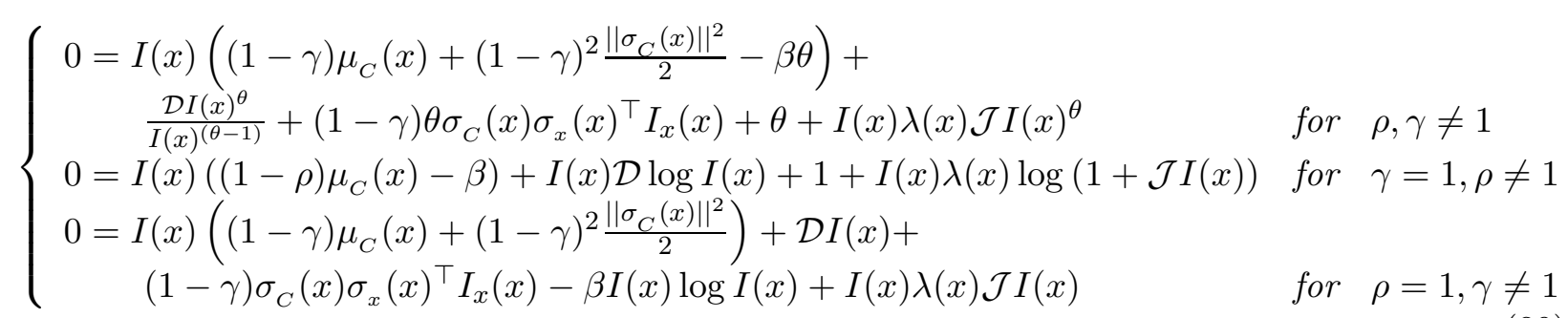

\footnotetext{
${ }^{14}$ Further discussion is provided in Chapter 10 of Duffie (2001).
} 
and satisfies the transversality condition $\left(\lim _{T \rightarrow \infty} \mathrm{E}[J(T)]=0\right.$ for $J(t)$ defined below) then the value function is given by:

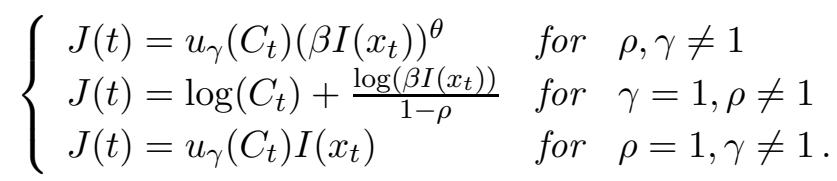

The corresponding pricing kernel is:

$$
\left\{\begin{array}{lll}
\Pi(t)=e^{-\int_{0}^{t}\left(\beta \theta+\frac{(1-\theta)}{I\left(x_{s}\right)}\right) d s}\left(C_{t}\right)^{-\gamma}\left(I\left(x_{t}\right)\right)^{(\theta-1)} & \text { for } & \rho, \gamma \neq 1 \\
\Pi(t)=e^{\left.-\int_{0}^{t} \frac{\beta}{I\left(x_{s}\right)}\right) d s} \frac{1}{\left(C_{t} I\left(x_{t}\right)\right)} & \text { for } & \gamma=1, \rho \neq 1 \\
\Pi(t)=e^{-\int_{0}^{t} \beta\left(1+\log I\left(x_{s}\right)\right) d s}\left(C_{t}\right)^{-\gamma} I\left(x_{t}\right) & \text { for } & \rho=1, \gamma \neq 1 .
\end{array}\right.
$$

Proof 1 We provide the proof for the case $\gamma, \rho \neq 1$. The special cases are treated similarly.

From its definition

$$
J(t)=\mathrm{E}_{t}\left(\int_{t}^{\infty} f\left(c_{s}, J(s)\right)\right)
$$

Thus, $J\left(x_{t}, c_{t}\right)+\int_{0}^{t} f\left(c_{s}, J\left(x_{s}, c_{s}\right)\right) d s$ is a martingale. This observation implies that:

$$
\mathrm{E}\left[d J\left(x_{t}, c_{s}\right)+f\left(c_{t}, J\left(x_{t}, c_{t}\right)\right) d t\right]=0 .
$$

Using our guess $\left(J(t)=u_{\gamma}\left(c_{t}\right) \beta^{\theta} I(x)^{\theta}\right)$ and applying the Itô-Doeblin formula we obtain:

$$
(1-\gamma) \mu_{c}\left(x_{t}\right)+(1-\gamma)^{2} \frac{\left\|\sigma_{c}\left(x_{t}\right)\right\|^{2}}{2}+\frac{\mathcal{D} I^{\theta}\left(x_{t}\right)}{I\left(x_{t}\right)^{\theta}}+\lambda(x) \mathcal{J} I(x)^{\theta}+\frac{\theta}{I\left(x_{t}\right)}-\beta \theta=0,
$$

where we have used the fact that

$$
\frac{f(c, J)}{J}=\frac{u_{\rho}\left(c_{t}\right)}{((1-\gamma) J)^{1 / \theta-1} J}-\beta \theta=\frac{\theta}{I(x)}-\beta \theta
$$

and the definition of the Dynkin operator $\mathcal{D} I(x)=I_{x}(x)^{\top} \mu_{x}(x)+\frac{1}{2} \operatorname{Trace}\left(I_{x x}(x) \sigma_{x}(x) \sigma_{x}(x)^{\top}\right)$. Rearranging we obtain the equation of the proposition.

Now suppose that $I(\cdot)$ solves this equation. Then, applying the Itô Doeblin formula to our candidate $J(t)$ we obtain

$$
\begin{aligned}
J(T) & =J(t)+\int_{t}^{T} \mathcal{D} J(s) d s+\int_{t}^{T} J_{c} \sigma_{c} d z_{c}(s)+\int_{t}^{T} J_{x} \sigma_{x} d z_{x}(s)+\int_{t}^{T} J\left(s^{-}\right)\left(\frac{I\left(X_{s^{-}}+\nu\right)^{\theta}}{I\left(X_{s^{-}}\right)^{\theta}}-1\right) d N(s) \\
& =J(t)-\int_{t}^{T} f\left(c_{s}, J_{s}\right) d s+\int_{t}^{T} J(s)(1-\gamma) \sigma_{c}\left(x_{s}\right) d z_{c}(s)+\int_{t}^{T} \theta J(s) \sigma_{I}\left(x_{s}\right) d z_{x}(s)+\int_{t}^{T} d M(s),(95)
\end{aligned}
$$

where we have defined $\sigma_{I}(x)=\frac{I^{\prime}(x)}{I(x)} \sigma_{x}(x)$ and the pure jump martingale

$$
M(t)=\int_{0}^{t} J\left(s^{-}\right)\left(\frac{I\left(X_{s^{-}}+\nu\right)^{\theta}}{I\left(X_{s^{-}}\right)^{\theta}}-1\right) d N(s)-\int_{0}^{t} \lambda\left(X_{s^{-}}\right) J\left(s^{-}\right) \mathcal{J} I\left(X_{s}\right)^{\theta} d s .
$$


If the stochastic integral is a martingale, ${ }^{15}$ and if the transversality condition is satisfied, then we obtain the desired result by taking expectations and letting $T$ tend to infinity:

$$
J(t)=\mathrm{E}\left[\int_{t}^{\infty} f\left(c_{s}, J_{s}\right) d s\right]
$$

which shows that our candidate $J(t)$ solves the recursive stochastic differential equation. Uniqueness follows (under some additional technical conditions) from the appendix in Duffie, Epstein, Skiadas (1992).

The next result investigates the property of equilibrium prices.

Proposition 2 The risk-free interest rate is given by:

$$
\left\{\begin{aligned}
r\left(x_{t}\right)= & \beta+\rho\left(\mu_{C}\left(x_{t}\right)+\frac{\left\|\sigma_{C}\left(x_{t}\right)\right\|^{2}}{2}\right)-\gamma(1+\rho) \frac{\left\|\sigma_{C}(x)\right\|^{2}}{2}- & & \\
& (1-\theta) \sigma_{I}(x)^{\top}\left(\sigma_{C}\left(x_{t}\right)+\frac{1}{2} \sigma_{I}\left(x_{t}\right)\right)+\lambda\left(x_{t}\right)\left(\frac{\theta-1}{\theta} \mathcal{J} I^{\theta}-\mathcal{J} I^{(\theta-1)}\right) & & \text { for } \quad \rho \neq 1 \\
r\left(x_{t}\right)= & \beta+\mu_{C}\left(x_{t}\right)+\frac{\left\|\sigma_{C}\left(x_{t}\right)\right\|^{2}}{2}-\gamma\left\|\sigma_{C}\left(x_{t}\right)\right\|^{2} & & \text { for } \quad \rho=1 .
\end{aligned}\right.
$$

Further, the value of the claim to aggregate consumption is given by:

$$
\left\{\begin{array}{ccc}
S(t)=C(t) I\left(x_{t}\right) & \text { for } & \rho \neq 1 \\
S(t)=\frac{C(t)}{\beta} & \text { for } & \rho=1 .
\end{array}\right.
$$

Thus

$$
\frac{d S_{t}}{S_{t}}=\mu_{S}\left(x_{t}\right) d t+\left(\sigma_{C}\left(x_{t}\right)+\sigma_{I}\left(x_{t}\right)\right) d z(t)+\nu_{I}\left(x_{t}\right) d N(t)
$$

where we have defined:

$$
\begin{aligned}
\sigma_{I}(x) & =\frac{1}{I(x)} I_{x}(x)^{\top} \sigma_{x}(x) \mathbf{1}_{\{\rho \neq 1\}} \\
\nu_{I}(x) & =\left(\frac{I(x+\widetilde{\nu})}{I(x)}-1\right) .
\end{aligned}
$$

The risk premium on the stock is given by

$\mu_{S}(x)+\frac{1}{I\left(x_{t}\right)}-r\left(x_{t}\right)=\left(\gamma \sigma_{C}\left(x_{t}\right)+(1-\theta) \sigma_{I}(x)\right)^{\top}\left(\sigma_{C}(x)+\sigma_{I}(x)\right)+\lambda\left(x_{t}\right)\left(\mathcal{J} I(x)^{\theta-1}-\mathcal{J} I(x)^{\theta}\right)$.

\footnotetext{
${ }^{15}$ Sufficient conditions are:$$
\mathrm{E}\left[\int_{0}^{T} J(s)^{2}\left(\sigma_{c}\left(x_{s}\right)^{2}+\sigma_{I}\left(x_{s}\right)^{2}\right) d s\right]<\infty \forall T>0
$$ 
Proof 2 To prove the result for the interest rate, apply Itô-Doeblin to the pricing kernel and it follows from $r(t)=-\mathrm{E}\left[\frac{d \Pi(t)}{\Pi(t)}\right] / d t$ that we obtain:

$$
r\left(x_{t}\right)=\beta \theta+\frac{(1-\theta)}{I\left(x_{t}\right)}+\gamma \mu_{c}\left(x_{t}\right)-\frac{1}{2} \gamma^{2}\left\|\sigma_{c}\left(x_{t}\right)\right\|^{2}-\frac{\mathcal{D} I\left(x_{t}\right)^{(\theta-1)}}{I\left(x_{t}\right)^{(\theta-1)}}-\lambda\left(X_{t}\right) \mathcal{J} I(x)^{\theta-1} .
$$

Now substitute the expression for $\frac{1}{I(x)}$ from the equation in (89) to obtain the result.

To prove the result for the consumption claim, define $S(t)=c_{t} I\left(x_{t}\right)$. Then using the definition of

$$
\Pi(t)=e^{-\beta \theta t-\int_{0}^{t} \frac{(1-\theta)}{I\left(x_{s}\right)} d s} c_{t}^{-\gamma} I\left(x_{t}\right)^{\theta-1}
$$

we obtain:

$$
d(\Pi(t) S(t))=e^{-\beta \theta t-\int_{0}^{t} \frac{(\theta-1)}{I\left(x_{s}\right)} d s}\left(d J(t)-J(t)\left(\beta \theta+\frac{(1-\theta)}{I\left(x_{t}\right)}\right) d t\right) .
$$

Now, note that by definition we have:

$$
\begin{aligned}
d J(t) & =-f\left(c_{t}, J\right) d t+d M_{t} \\
& =-J(t)\left(\frac{\theta}{I\left(x_{t}\right)}-\theta \beta\right) d t+d M_{t}
\end{aligned}
$$

for some P-martingale M. Combining this observation with (104), we get:

$$
\begin{aligned}
d(\Pi(t) S(t)) & =e^{-\beta \theta t-\int_{0}^{t} \frac{(1-\theta)}{I\left(x_{s}\right)} d s} \frac{(-J(t))}{I\left(x_{t}\right)} d t+e^{-\beta \theta t-\int_{0}^{t} \frac{(1-\theta)}{I\left(x_{s}\right)} d s} d M_{t} \\
& =-\Pi(t) c(t) d t+e^{-\beta \theta t-\int_{0}^{t} \frac{(1-\theta)}{I\left(x_{s}\right)} d s} d M_{t} .
\end{aligned}
$$

Thus integrating we obtain

$$
\Pi(T) S(T)+\int_{t}^{T} \Pi(s) c_{s} d s=\Pi(t) S(t)+\int_{t}^{T} e^{-\beta \theta(u-t)-\int_{t}^{u} \frac{(1-\theta)}{I\left(x_{s}\right)} d s} d M_{u} .
$$

Taking expectations and letting $T \rightarrow \infty$ and assuming the transversality condition holds (i.e., $\lim _{T \rightarrow \infty} \mathrm{E}[\Pi(T) S(T)]=0$ ), we obtain the desired result:

$$
\Pi(t) S(t)=\mathrm{E}_{t}\left[\int_{t}^{\infty} \Pi(s) c_{s} d s\right] .
$$

\section{Appendix B: The Price of an Option in the Post-Crash Affine Model}

We note that the model in Section 2 is affine. In particular, the value of the dividend claim $V_{t}=D_{t} e^{F+G x_{t}}$ has the following risk-neutral dynamics: 
$\frac{d V_{t}}{V_{t}}=\left(\mu_{0}^{Q}+\mu_{1}^{Q} x_{t}\right) d t+\sigma_{D} \sqrt{\Omega}\left(\rho_{C, D} d z_{c}^{Q}(t)+\sqrt{1-\rho_{C, D}^{2}} d z_{D}^{Q}(t)\right)+G \sigma_{x} \sqrt{\Omega} d z_{x}^{Q}(t)+\left(e^{G \tilde{\nu}}-1\right) d N(t)$,

where

$$
\begin{aligned}
\mu_{0}^{Q} & =\mu_{D}-\gamma \rho_{C, D} \sigma_{D} \Omega-(1-\theta) B \sigma_{x}^{2} \Omega G+\frac{1}{2} \sigma_{x}^{2} \Omega G^{2} \\
\mu_{1}^{Q} & =\phi-\kappa_{x} G .
\end{aligned}
$$

As such, the option pricing problem can be solved using standard inverse Fourier transform techniques. In particular, the date- $t$ value of a European call option on the dividend claim $V_{t}$, with maturity $T$ and strike price $K$, is given by

$$
\begin{aligned}
C\left(V_{t}, x_{t}, K, T\right) & =\mathrm{E}_{t}^{Q}\left[e^{-\int_{t}^{T} d s r_{s}}\left(V_{T}-K\right) \mathbf{1}_{\left\{V_{T}>K\right\}}\right] \\
& =\Psi_{t, 1}(\log K)-K \Psi_{t, 0}(\log K)
\end{aligned}
$$

where we have defined:

$$
\Psi_{t, a}(k) \equiv \mathrm{E}_{t}^{Q}\left[e^{-\int_{t}^{T} r_{s} d s} e^{a \log V_{T}} \mathbf{1}_{\left\{\log V_{T}>k\right\}}\right]
$$

Following Bates (1996), Heston (1993), Duffie et al. (2000), and others, we use the Fourier inversion theorem for the random variable $\left(\log V_{t}\right)$ to obtain:

$$
\Psi_{t, a}(k)=\frac{\psi_{t}(a)}{2}+\frac{1}{\pi} \int_{0}^{\infty} \frac{\mathcal{I M}\left[\psi_{t}(a+\mathcal{I} v) e^{-\mathcal{I} v k}\right]}{v} d v
$$

In equation (113), the transform $\psi_{t}(\alpha) \equiv \mathrm{E}_{t}^{Q}\left[e^{-\int_{t}^{T} r_{s} d s} e^{\alpha \log V_{T}}\right]$ admits the following closed form solution:

$$
\psi_{t}(\alpha)=\exp \left(M(T-t)+N(T-t) x_{t}+\alpha \log V_{t}\right),
$$

where the functions $M(\tau), N(\tau)$ satisfy

$$
\begin{aligned}
& N(\tau)=\left(\alpha \mu_{1}^{Q}-\rho\right)\left(\frac{1-e^{-\kappa_{x} \tau}}{\kappa_{x}}\right) \\
& M(\tau)=\int_{0}^{\tau} d \tau\left\{\frac{N^{2} \sigma_{x}^{2} \Omega}{2}-N(1-\theta) B \sigma_{x}^{2} \Omega+\alpha \mu_{0}^{Q}-r_{0}+\alpha(\alpha-1) \frac{\Omega\left(\sigma_{D}^{2}+G^{2} \sigma_{x}^{2}\right)}{2}+\lambda^{Q}\left(\chi_{N+\alpha G}^{Q}-1\right)\right\} .
\end{aligned}
$$


Proof 3 The proof simply consists in showing that $\left[e^{-\int_{0}^{t} r_{s} d s} \exp \left(M(T-t)+N(T-t) x_{t}+\alpha \log V(t)\right)\right]$ is a $Q$-martingale. Indeed, in that case

$$
\begin{aligned}
e^{-\int_{0}^{t} r_{s} d s} \exp \left(M(T-t)+N(T-t) x_{t}+\alpha \log V(t)\right) & =\mathrm{E}^{Q}\left[e^{-\int_{0}^{T} r_{s} d s} \exp \left(M(0)+N(0) x_{t}+\alpha \log V(T)\right)\right] \\
& =\mathrm{E}^{Q}\left[e^{-\int_{0}^{T} r_{s} d s} \exp (\alpha \log S(T))\right] \\
& =e^{-\int_{0}^{t} r_{s} d s} \psi_{t}(\alpha)
\end{aligned}
$$

which is the desired result. To verify the martingale condition we apply Itô-Doeblin formula to

$$
Y_{t} \equiv e^{-\int_{0}^{t} r_{s} d s} \exp \left(M(T-t)+N(T-t) x_{t}+\alpha \log V(t)\right)
$$

and obtain that

$$
\mathrm{E}_{t}\left[d Y_{t}\right]=0
$$

holds when $N$ and $M$ satisfy equations (115)-(116) above. A standard argument then shows that $Y_{t}$ is a $Q$-martingale.

\section{References}

Abel, Andrew B., 1999, Risk Premia and Term Premia in General Equilibrium, Journal of Monetary Economics 43, 3-33.

Ahn, Chang Mo, and Howard E. Thompson, 1988, Jump-Diffusion Processes and the Term Structure of Interest Rates Journal of Finance 43, 155-174.

Attanasio, Orazio P., and Guglielmo Weber, 1989, Intertemporal Substitution, Risk Aversion and the Euler Equation for Consumption, Economic Journal 99, 59-73.

Bakshi, Gurdip, Charles Cao, and Zhiwu Chen, 1997, Empirical Performance of Alternative Option Pricing Models, Journal of Finance 52, 2003-2049.

Bakshi, Gurdip, Charles Cao, and Zhiwu Chen, 2000, Pricing and Hedging Long-Term Options, Journal of Econometrics 94, 277-318.

Bakshi, Gurdip, and Nikunj Kapadia, 2003, Delta-Hedged Gains and the Negative Market Volatility Risk Premium, Review of Financial Studies 16, 527-566.

Bakshi, Gurdip, Nikunj Kapadia, and Dilip Madan, 2003, Stock Return Characteristics, Skew Laws, and the Differential Pricing of Individual Equity Options, Review of Financial Studies $16,101-143$. 
Bansal, Ravi, and Christian Lundblad, 2002, Market Efficiency, Asset Returns, and the Size of the Risk Premium in Global Equity Markets, Journal of Econometrics 109, 195-237.

Bansal, Ravi, and Amir Yaron, 2004, Risks for the Long Run: A Potential Resolution of Asset Pricing Puzzles, Journal of Finance 59, 1481-1509.

Barro, Robert J., 2005, Rare Events and the Equity Premium, Working Paper, Harvard University.

Bates, David S., 1996, Jumps and Stochastic Volatility: Exchange Rate Processes Implicit in Deutsche Mark Options, Review of Financial Studies 1, 69107.

Bates, David S., 2000, Post-'87 Crash Fears in the S\&P 500 Futures Option Market, Journal of Econometrics 94, 181-238.

Bates, David S., 2001, The Market for Crash Risk, Working Paper, University of Iowa.

Benzoni, Luca, 2002, Pricing Options under Stochastic Volatility: An Empirical Investigation, Working Paper, University of Minnesota.

Bollen, Nicolas P. B., and Robert E. Whaley, 2004, Does Net Buying Pressure Affect the Shape of Implied Volatility Functions? Journal of Finance 59, 711-753.

Bondarenko, Oleg, 2003, Why are Put Options So Expensive?, Working Paper, University of Illinois at Chicago.

Broadie, Mark, Mikhail Chernov, and Michael S. Johannes, 2004, Specification and Risk Premiums: The Information in S\&P 500 Futures Options, Working Paper, Columbia GSB.

Brown, David P., and Jens C. Jackwerth, 2004, The Pricing Kernel Puzzle: Reconciling Index Option Data and Economic Theory, Working Paper, UW Madison and University of Konstanz.

Buraschi, Andrea, and Jens Jackwerth, 2001, The Price of a Smile: Hedging and Spanning in Option Markets, Review of Financial Studies 14, 495-527.

Buraschi, Andrea, and Alexei Jiltsov, 2005, Option Volume and Difference in Beliefs, Journal of Finance, forthcoming.

Campbell, John Y., and John H. Cochrane, 1999, By Force of Habit: A Consumption-Based Explanation of Aggregate Stock Market Behavior, Journal of Political Economy 107, 205251. 
Campbell, John Y., and Robert J. Shiller, 1988, The Dividend-Price Ratio and Expectations of Future Dividends and Discount Factors, Review of Financial Studies 1, 195-228.

Chacko, George, and Luis M. Viceira, 2003, Spectral GMM Estimation of Continuous-Time Processes, Journal of Econometrics 116, 259-292.

Chen, Long, Pierre Collin-Dufresne, and Robert S. Goldstein, 2005, On the Relation Between Credit Spread Puzzles and the Equity Premium Puzzle, Working Paper, Michigan State University, UC Berkeley, and University of Minnesota.

Chernov, Mikhail, A. Ronald Gallant, Eric Ghysels, and George Tauchen, 1999, A New Class of Stochastic Volatility Models with Jumps: Theory and Estimation, Working Paper, Columbia GSB, Duke, and University of North Carolina.

Chernov, Mikhail, A. Ronald Gallant, Eric Ghysels, and George Tauchen, 2003, Alternative Models for Stock Price Dynamics, Journal of Econometrics 116, 225-257.

Chernov, Mikhail, and Eric Ghysels, 2000, A Study Towards a Unified Approach to the Joint Estimation of Objective and Risk Neutral Measures for the Purpose of Options Valuation, Journal of Financial Economics 56, 407-458.

Collin-Dufresne, Pierre, and Robert S. Goldstein, 2005, Improving on the Campbell-Shiller LogLinearization Approximation, Working Paper, UC Berkeley and University of Minnesota.

Collin-Dufresne, Pierre, Robert S. Goldstein, and Jean Helwege, 2003, Is Credit Event Risk Priced? Modeling Contagion via the Updating of Beliefs, Working Paper, UC Berkeley, University of Minnesota, and University of Arizona.

Constantinides, George M., Jens C. Jackwerth, and Stylianos Perrakis, 2004, Mispricing of S\&P 500 Index Options, Working Paper, University of Chicago, University of Konstanz, and Concordia University.

Coval, Joshua D., and Tyler Shumway, 2001, Expected Option Returns, Journal of Finance 56, 983-1009.

Cvitanić, Jakša, Vassilis Polimenis, and Fernando Zapatero, 2005, Optimal Portfolio Allocation with Higher Moments, Working Paper, University of Southern California and UC Riverside.

Dennis, Patrick, and Stewart Mayhew, 2002, Risk-Neutral Skewness: Evidence from Stock Options, Journal of Financial and Quantitative Analysis 37, 471-493. 
Driessen, Joost, and Pascal Maenhout, 2004, A Portfolio Perspective on Option Pricing Anomalies, Working Paper, University of Amsterdam amd INSEAD.

Duffie, Darrell, 2001, Dynamic Asset Pricing Theory, Princeton University Press, Princeton and Oxford.

Duffie, Darrell, and Larry G. Epstein, 1992a, Stochastic Differential Utility, Econometrica 60, 353-394.

Duffie, Darrell, and Larry G. Epstein, 1992b, Asset Pricing with Stochastic Differential Utility, Review of Financial Studies 5, 411-436.

Duffie, Darrell, Jun Pan, and Kenneth Singleton, 2000, Transform Analysis and Asset Pricing for Affine Jump Diffusions, Econometrica 68, 1343-137.

Duffie, Darrell, and Costis Skiadas, 1994, Continuous-Time Security Pricing: A Utility Gradient Approach, Journal of Mathematical Economics 23, 107-132.

Epstein, Larry G., and Stanley E. Zin, 1989, Substitution, Risk Aversion, and the Temporal Behavior of Consumption and Asset Returns: A Theoretical Framework, Econometrica 57, 937-969.

Eraker, Bjørn, 2004, Do Stock Prices and Volatility Jump? Reconciling Evidence from Spot and Option Prices, Journal of Finance 59, 1367-1404.

Eraker, Bjørn, Michael Johannes, and Nicholas Polson, 2003, The Impact of Jumps in Volatility and Returns Journal of Finance 58, 1269-1300.

Figlewski, Stephen, 1989, Options Arbitrage in Imperfect Markets, Journal of Finance 44, 12891311.

Fisher, Mark, and Christian Gilles, 1999, Consumption and Asset Prices with Homothetic Recursive Preferences, Working Paper, Federal Reserve Bank of Atlanta.

Foresi, Silverio, and Liuren Wu, 2003, Crash-O-Phobia: A Domestic Fear or A Worldwide Concern? Working Paper, Baruch College and The Goldman Sachs Group, Inc..

Gârleanu, Nicolae B., Lasse H. Pedersen, and Allen M. Poteshman, 2005, Demand-Based Option Pricing, Working Paper, University of Pennsylvania, NYU, and UIUC. 
Green, T. Clifton, and Stephen Figlewski, 1999, Market Risk and Model Risk for a Financial Institution Writing Options Journal of Finance 54, 1465-1499.

Guvenen, Fatih, 2001, Mismeasurement of the Elasticity of Intertemporal Substitution: The Role of Limited Stock Market Participation. Working Paper, University of Rochester.

Hall, Robert E., 1988, Intertemporal Substitution in Consumption Journal of Political Economy 96, 339-357.

Han, Bing, 2005, Limits of Arbitrage, Sentiment and Pricing Kernel: Evidences from Index Options, Working Paper, Ohio State University.

Hansen, Lars P., John C. Heaton, and Nan Li, 2004, Consumption Strikes Back, Working Paper, University of Chicago.

Hansen, Lars P., and Kenneth J. Singleton, 1982, Generalized Instrumental Variables Estimation of Nonlinear Rational Expectations Models, Econometrica 50, 1269-1286.

Heston, Steven L., 1993, A Closed-Form Solution for Options with Stochastic Volatility with Applications to Bond and Currency Options, Review of Financial Studies 6, 327-343.

Huang, Jing-zhi, and Liuren Wu, 2004, Specification Analysis of Option Pricing Models Based on Time-Changed Levy Processes, Journal of Finannce 59, 1405-1440.

Hugonnier, Julien, Dmitry Kramkov, and Walter Schachermayer, 2005, On Utility Based Pricing of Contingent Claims in Incomplete Markets, Mathematical Finance 15, 203-212.

Jackwerth, Jens C., 2000, Recovering Risk Aversion from Option Prices and Realized Returns, Review of Financial Studies 13, 433-451.

Jackwerth, Jens C., and Mark Rubinstein, 1996, Recovering Probability Distributions from Option Prices, Journal of Finance 51, 1611-1631.

Jones, Christopher S., 2003, The Dynamics of Stochastic Volatility: Evidence from Underlying and Options Markets, Journal of Econometrics 116, 181-224.

Kleidon, Allan W., 1992, Arbitrage, Nontrading, and Stale Prices: October 1987, Journal of Business 65, 483-507.

Kreps, David M., and Evan L. Porteus, 1978, Temporal Resolution of Uncertainty and Dynamic Choice Theory, Econometrica 46, 185-200. 
Liu, Jun, and Francis A. Longstaff, 2004, Losing Money on Arbitrage: Optimal Dynamic Portfolio Choice in Markets with Arbitrage Opportunities, Review of Financial Studies 17, 611-641.

Liu, Jun, Francis A. Longstaff, and Jun Pan, 2003, Dynamic Asset Allocation with Event Risk, Journal of Finannce 58, 231-259.

Liu, Jun, and Jun Pan, 2003, Dynamic Derivative Strategies, Journal of Financial Economics 69, 401-430.

Liu, Jun, Jun Pan, and Tan Wang, 2005, An Equilibrium Model of Rare-Event Premia and Its Implication for Option Smirks, Review of Financial Studies 18, 131-164.

Longstaff, Francis A., 1995, Option Pricing and the Martingale Restriction, Review of Financial Studies 8, 1091-1124.

Malloy, Christopher J., Tobias J. Moskowitz, and Annette Vissing-Jorgensen, 2005, Job Risk and Asset Returns, Working Paper, London Business School, University of Chicago, and Northwestern University.

Mehra, Rajnish, and Edward C. Prescott, 1985, The Equity Premium: A Puzzle, Journal of Monetary Economics 15, 145-161.

Naik, Vasant, and Moon Lee, 1990, General Equilibrium Pricing of Options on the Market Portfolio with Discontinuous Returns, Review of Financial Studies 3, 493-521.

Pan, Jun, 2002, The Jump-Risk Premia Implicit in Options: Evidence from an Integrated TimeSeries Study, Journal of Financial Economics 63, 3-50.

Rosenberg, Joshua V., and Robert F. Engle, 2002, Empirical Pricing Kernels, Journal of Financial Economics 64, 341-372.

Rubinstein, Mark, 1994, Implied Binomial Trees, Journal of Finannce 49, 771-818.

Santa-Clara, Pedro, and Alessio Saretto, 2004, Option Strategies: Good Deals and Margin Calls, Working Paper, UCLA.

Schroder, Mark, and Costis Skiadas, 1999, Optimal Consumption and Portfolio Selection with Stochastic Differential Utility, Journal of Economic Theory 89, 68-126. 
Schroder, Mark, and Costis Skiadas, 2003, Optimal Lifetime Consumption-Portfolio Strategies under Trading Constraints and Generalized Recursive Preferences, Stochastic Processes and their Applications 108, 155-202.

Shephard, Neil G., Andrew C. Harvey, 1990, On the Probability of Estimating a Deterministic Component in the Local Level Model, Journal of Time Series Analysis 11, 339-347.

Shleifer, Andrei, and Robert W. Vishny, 1997, The Limits of Arbitrage, Journal of Finannce 52, $35-55$.

Skiadas, Costis, 2003, Robust Control and Recursive Utility, Finance and Stochastics 7, 475-489. 